\title{
Intramolecular Enolate Alkylation: From Steroids through Cladiellins to Isolaurallene
}

\author{
Deukjoon Kim \\ College of Pharmacy, Seoul National University, Seoul 151-742, Republic of Korea \\ Fax+82(2)8880649; E-mail: deukjoon@snu.ac.kr \\ Received: 20.08.2013; Accepted after revision: 24.09.2013
}

On the eve of my retirement, it is my great pleasure to dedicate this account to Professors Steven Weinreb and Gilbert Stork for their insightful tutelage and inspiration throughout my career.

\begin{abstract}
This account of the author's contributions over his career attempts to sketch out a research arc that stretches all the way from very simple beginnings, taking inspiration from the steroidal transhydrindane problem, to the development of a general strategy for the stereoselective construction of cycloalkanecarboxylates via a folding and allylic strain controlled intramolecular ester enolate alkylation, with applications thereof to the total syntheses of natural products of modest complexity. Examination of the corresponding $\mathrm{S}_{\mathrm{N}} 2^{\prime}$ version of this methodology led to a serendipitous discovery that ultimately produced the olefin geometry dependent intramolecular amide enolate alkylation. Applications and extensions of this methodology have enabled completely substrate-controlled asymmetric total syntheses of diverse medium-ring oxacyclic marine natural products, and a fortuitous discovery along the way involving an organoselenium-based method led to an intriguing biomimetic synthesis of Laurencia metabolites. Observations are made regarding aspects of a research career in retrospect.
\end{abstract}

\footnotetext{
1 Introduction

2 Intramolecular $\mathrm{S}_{\mathrm{N}} 2$ Enolate Alkylation

3 Intramolecular $\mathrm{S}_{\mathrm{N}} 2^{\prime}$ Enolate Alkylation

4 Synthesis of $\alpha, \alpha^{\prime}$-cis-Disubstituted Medium-Ring OxaSynthesis of $\alpha, \alpha^{\prime}$-cis-Disubstitut
cyclic Marine Natural Products

5 Synthesis of $\alpha, \alpha^{\prime}$-trans-Disubstituted Medium-Ring Oxacyclic Marine Natural Products

6 General Synthetic Plan for Dioxabicyclic Bromoallene Marine Natural Products Having either a 2,10-Dioxabicyclo[7.3.0]dodecene or 2,9-Dioxabicyclo[6.3.0]undecene Skeleton

7 Conclusion
}

Key words: intramolecular enolate alkylation, natural products, substrate-controlled synthesis, medium-ring ethers, ring-closing metathesis

\section{$1 \quad$ Introduction}

Owing to their physiological activities and esthetic molecular architecture, steroids, such as cortisone (1) (Scheme 1), have played a very important role in the development of new synthetic methodologies, notably exemplified by Gilbert Stork's work in this area. In particular, the thermodynamically less stable trans-fused CD ring system (the so-called trans-hydrindane) found in most steroids has served as an attractive and challenging target for synthetic organic chemists.

SYNLETT 2014, 25, 0033-0057

Advanced online publication: 12.11.2013

DOI: 10.1055/s-0033-1340050; Art ID: ST-2013-A0802-A

(C) Georg Thieme Verlag Stuttgart · New York
Predictably, Stork's predilection for important synthesis problems influenced me when I started my independent research in the early 1980s at the College of Pharmacy, Seoul National University, and I embarked on a novel stereoselective construction of the trans-hydrindane system. I envisioned that the intramolecular ester enolate alkylation (IEEA) of tosylate $\mathbf{2}$ might produce cis-1,2-dialkylcyclohexanecarboxylate $\mathbf{3}$ in a stereoselective manner with the creation of a quaternary carbon center, probably through 'H-eclipsed' transition state geometry $\mathbf{A}$, as rationalized on basis of the allylic strain concept. This product hardly looks like a trans-hydrindane precursor; the $n$-butyl side chain does not have any functionality necessary for forming the five-membered $\mathrm{D}$ ring, not to mention the cyclohexane $\mathrm{C}$ ring having nothing from which to grow out the $\mathrm{A}$ and $\mathrm{B}$ rings.

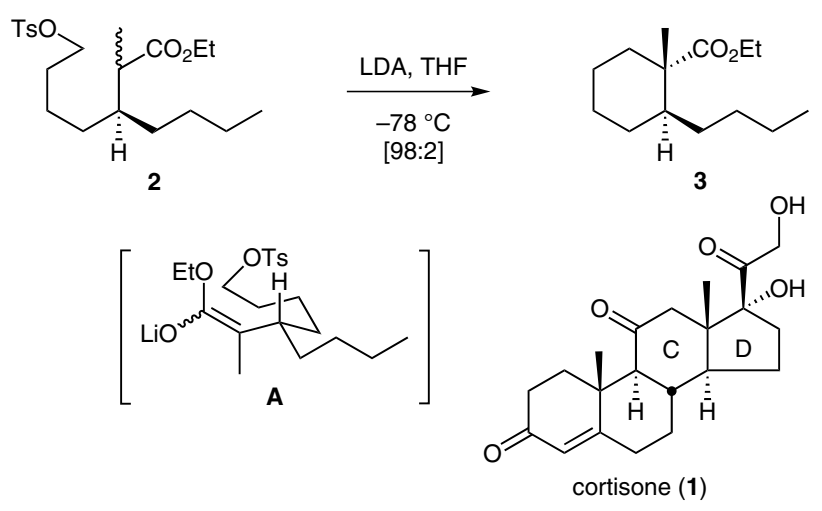

Scheme 1 Approach to steroidal trans-hydrindanes via intramolecular ester enolate alkylation

Resources in the laboratory were sparse at the time, and the only organometallic reagent we had was a bottle of $n$ butyllithium $(n$-BuLi); this we used to introduce the $n$-butyl side chain and to generate lithium diisopropylamide (LDA). When I visited Professor Eiichi Nakamura at the Tokyo Institute of Technology (now at the University of Tokyo), he arranged for me to meet Professor Jiro Tsuji. While visiting his office, Tsuji told me, "Please take any equipment or reagent that you need from my laboratories back with you", which overwhelmed and thrilled me. I took one of many shiny brand-new Schlenk flasks in his 
laboratories back home, and used that memorable glassware to generate the LDA solution.

Our work was published in Tetrahedron Letters in 1986 and became the first publication of my independent career. ${ }^{1}$ Apparently, the reviewers recognized the synthetic potential of our IEEA methodology despite the drawbacks discussed above. I had never had any doubts that the IEEA would work and could be developed into a viable methodology. That was because when I showed it to him on the blackboard in his office, Gilbert Stork told me it was a good idea.

\section{Intramolecular $S_{N} 2$ Enolate Alkylation}

Since our early work, we have examined the scope of our intramolecular enolate alkylation methodology in terms of ring size and other parameters within the context of natural product total synthesis, with particular emphasis on stereo-, regio-, and chemoselectivity. This effort has been well rewarded.

By the following year of our initial work, we had accomplished an efficient synthesis of geijerone (4) based on an ortho ester Claisen rearrangement/IEEA strategy (Scheme 2). ${ }^{2}$ It is plain to see from the enhanced sophistication in the synthetic intermediate that by that time the laboratory was better funded and we had more chemicals and equipment.

Our manuscript describing this work was submitted to Tetrahedron Letters, but was rejected on the grounds that it represented merely an extension of our previously reported work - I had no convincing counterargument to that! We then submitted the same manuscript to the Journal of Organic Chemistry as a communication. I cannot recall my rationale for such a move, but in the event it was accepted with minor revision.

A complicating factor was that I had already applied for a grant with the 'proposed' synthesis of geijerone, since I anticipated the manuscript would be rejected again. At that time, it was the customary grantsmanship strategy to

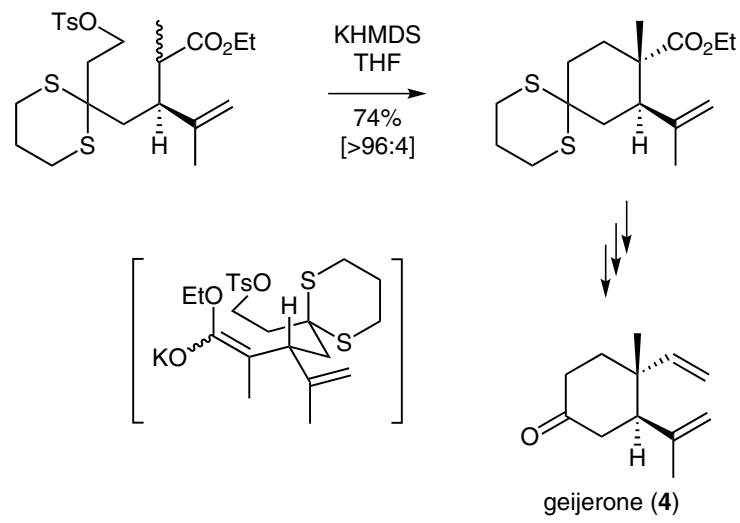

Scheme 2 Intramolecular ester enolate alkylation in the synthesis of geijerone (4)

write up a proposal covering work that had been more or less already completed. That way, one could publish the results soon after the grant was approved, and then start a new project with the funding. However, if the communication was published before a decision was reached on my grant proposal, I reasoned that I might need to explain the embarrassing scenario.

I called the late Professor Werner Herz, my editor, with the peculiar request that he delay the publication of our communication. I might add that international telephone calls at that time were still very expensive. He told me, "If you don't mind my saying this, the whole thing sounds a bit silly to me. Everyone else is calling me to facilitate publication, not to delay it. It is supposed to be a communication for rapid publication."

Around that time, the late Professor Barton visited Seoul for an international conference. Most of the Korean speakers did not have much to talk about on their independent research, and we were allocated about 15 minutes each. After my talk, Professor Barton told me, "You spoke very well, but you should synthesize something that is biologically active." I replied, "If I do that, it is highly likely that I will get scooped every time." He told me then,

\section{Biographical Sketch}

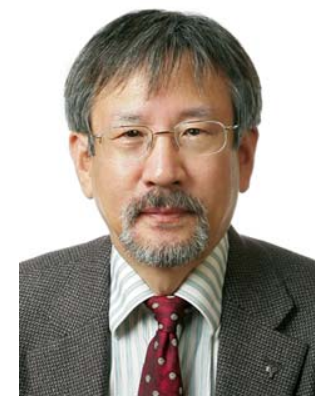

Deukjoon Kim was born in 1948 in Pusan, Korea, and graduated from the College of Pharmacy, Seoul National University, in 1970. He received his doctoral degree in chemistry from the Department of Chemistry, Fordham University, USA, under the mentorship of Professor Steven M. Weinreb, and carried out postdoctoral work under the guidance of Professor Gilbert Stork at the Department of Chemistry, Columbia University. He joined the faculty of the College of Pharmacy, Seoul National University, in 1983 and retired from there on August 31,2013 . His research interests are focused on the total synthesis of structurally complex natural products, especially those having bio- logical activity. He was elected to the Korean Academy of Science and Technology in 1999, was an invited lecturer at the 2008 Natural Products Gordon Research Conference (GRC), and was the recipient of the 2011 Korean Academy of Science and Technology Award. 
"Even so, you should try to synthesize a biologically active molecule."

I followed his advice, and the first synthetic target having both decent structural complexity and potent biological activity which captured my attention at this early stage of my career was (-)-fumagillol (5), a saponification product of the antiangiogenic fumagillin. We were intrigued by the possibility that an intramolecular glycolate enolate alkylation, as summarized in Scheme $3(\mathbf{6} \rightarrow \mathbf{7}$, the ratio given in square brackets is the ratio of isomeric products), might be utilized to help install the spirocyclic epoxide functionality in fumagillol in a stereoselective fashion. One of the students involved in this first asymmetric total synthesis of fumagillol ${ }^{3}$ continued working in this area at a pharmaceutical company, and has recently developed a semisynthetic analogue from fermented fumagillol which is undergoing clinical trials. It seems that in the end, I accomplished something that the late Professor Barton would consider useful, albeit in a vicarious manner.<smiles>C=CCC=C(C)C(CC(C)=O)C(CC(=O)O)C([18O])[18O]</smiles>

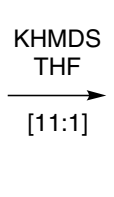

$\mathrm{BnO}$
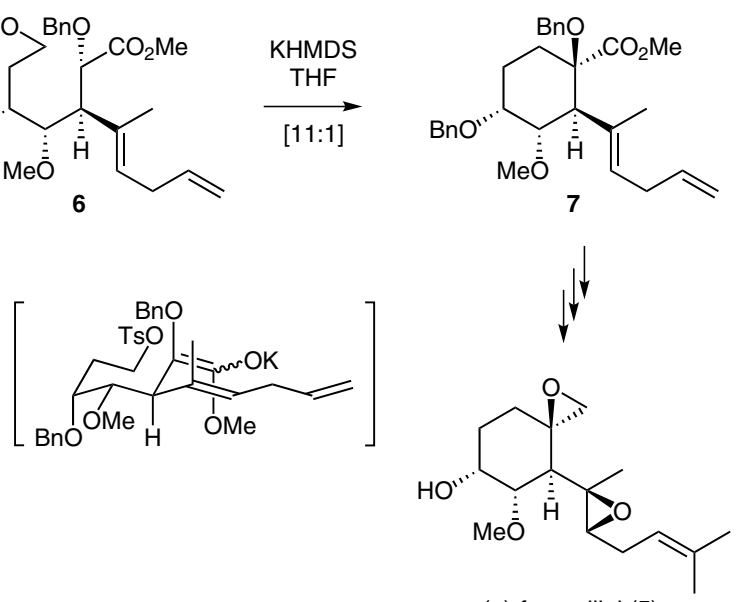

(-)-fumagillol (5)
Scheme 3 First asymmetric total synthesis of fumagillol (5) via intramolecular glycolate enolate alkylation

We successfully applied our folding and allylic strain controlled IEEA methodology to the total synthesis of natural products that possess a cyclopentane nucleus with a diverse substitution pattern. Furthermore, our synthesis of oppositol (8), outlined in Scheme 4, touched on a different perspective in the sense that the IEEA strategy $(\mathbf{9} \rightarrow \mathbf{1 0})$ was elaborated to establish the relative stereochemistry of the three contiguous stereogenic centers in the cyclopentane nucleus of the natural product in a single operation. ${ }^{4}$ This was accomplished using a doubly diastereodifferentiating, folding and allylic strain controlled IEEA in which the preferred H-eclipsed ester enolate B with its bulky side chain appendage in an equatorial position distinguishes the two homomorphic diastereotopic bromoethyl groups.

At a research meeting during my time at Columbia, Gilbert Stork mentioned in passing an example in which he employed a doubly diastereodifferentiating process which was disclosed in a US patent in the late 1950s (!). ${ }^{5}$ This

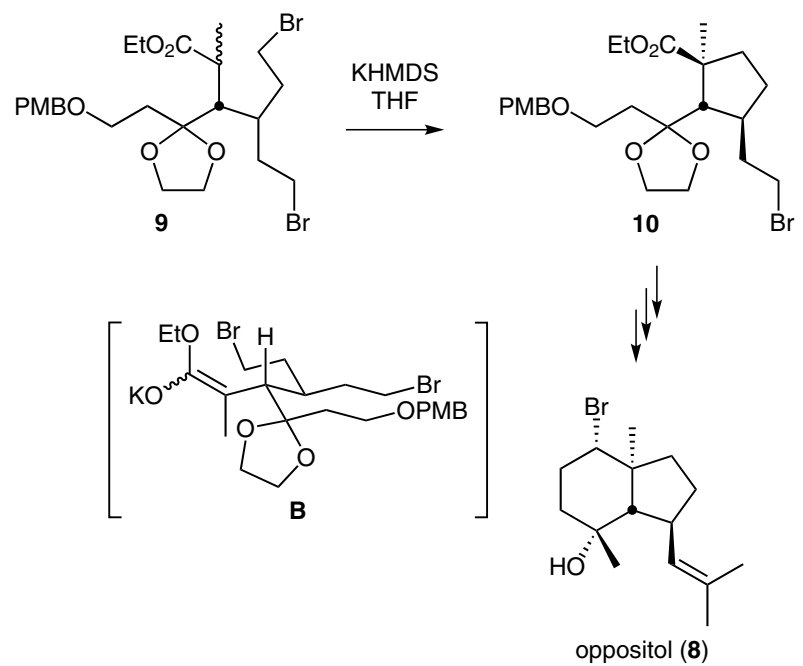

Scheme 4 Synthesis of oppositol (8) via doubly diastereodifferentiating, folding and allylic strain controlled intramolecular ester enolate alkylation

awesome chemistry done at such an early date made a deep impression on me, and I have no doubt it was the inspiration for our key step.

In 1984, Professor Takashi Tokoroyama in Japan introduced the terminology 'folding strain control' to elaborate on the diastereoselectivity observed during intramolecular cyclization reactions, ${ }^{6}$ and our IEEA reactions are fine examples of this concept. In fact, Tokoroyama systematically investigated the diastereoselectivity of our IEEA methodology for the construction of cyclohexanecarboxylates with additional substituents at various positions in the chain in connection with the concept of folding strain control, and in 1996 he and Kusaka published a full paper entitled Folding Strain Stereocontrol in Cyclohexane Ring Formation by Means of an Intramolecular Ester Enolate Alkylation Reaction.?

I must confess that in the past, I would deliberately describe our examples of intramolecular enolate alkylations as folding and allylic strain controlled in part for a nonscientific reason: it was a shrewd gamble that our manuscript might go to Tokoroyama for review! To the best of my knowledge, I was the only one besides him who actively used the phrase 'folding strain controlled'. When I gave a talk at the $2^{\text {nd }}$ Korea-USA Joint Symposium on Organic Chemistry in Seoul, I recall referring to such situations as 'chemopolitics', and this seemed to strike a resonant chord with the audience, in particular among the American delegation. Incidentally, this group included about a dozen eminent organic chemists, and this had a huge impact on the Korean organic synthesis community, but unfortunately the symposium was discontinued afterwards owing to budgetary problems. The postsymposium celebration was a highly memorable event in true 'Gangnam Style', well before Psy managed to acquaint the rest of the world with that way of life.

Owing to its remarkable biological activity, grandisol (14) (Scheme 5), a sex attractant of the male boll weevil $\mathrm{An}$ - 
thonomus grandis Boheman, has served as a testing ground to evaluate strategies for the stereoselective construction of four-membered rings, as exemplified by Stork's epoxy nitrile cyclization. ${ }^{8}$ In addition, the diastereomer fragranol (11) has been isolated from the roots of Artemisia fragrans Willd. Obviously, we were interested in whether our folding and allylic strain controlled IEEA strategy would be viable in the case of four-membered ring synthesis. ${ }^{9}$

This study illustrated a general trend in our methodology; namely, the larger the size of the $\alpha$-substituent group (R), the more inferior the stereoselectivity, as exemplified by the isomeric ratios of 19:1 and approximately 2:1 achieved for the intramolecular alkylation of an $\alpha$-methylsubstituted ester $(\mathbf{1 2} \rightarrow \mathbf{1 3})$ and the corresponding $\alpha$-allylsubstituted derivative $(\mathbf{1 5} \rightarrow \mathbf{1 6})$, respectively (Scheme $5)$. The inferior stereoselectivity observed in the transformation of allyl derivative $\mathbf{1 5}$ into cyclobutane 16, a step in the potential synthesis of grandisol (14), was overcome using the intramolecular alkylation of a cyclic enolate $-\mathrm{a}$ $\gamma$-lactone enolate $(\mathbf{1 7} \rightarrow \mathbf{1 8})$.

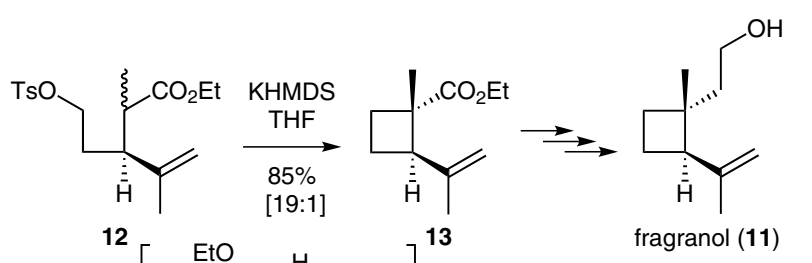

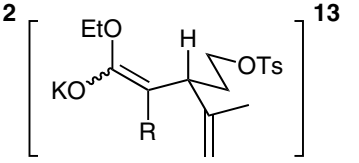<smiles>C=CC[C@H](CCO[Na])[C@@H](CCC)C(=C)C</smiles>

15

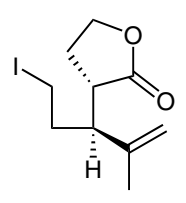

17

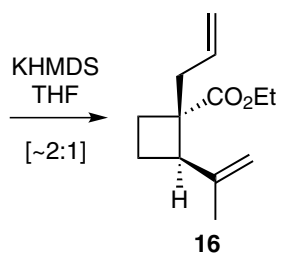

16

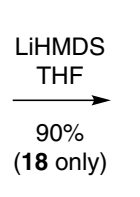

(18 only)

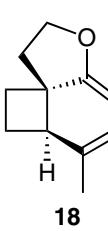

18

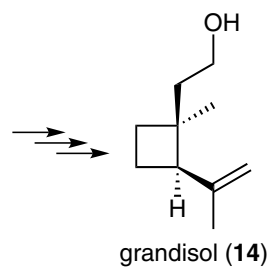

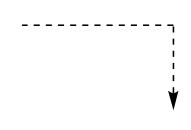

Scheme 5 Synthesis of grandisol (14) via intramolecular lactone enolate alkylation

I would like to digress for a while to describe a background story about our brefeldin synthesis, which was published in $1995 .{ }^{10}$ Under normal circumstances, I would not have been interested in a total synthesis of brefeldin A (19), simply because its cyclopentane nucleus does not possess a quaternary center. However, my casual curiosity about the comparative stereoselectivity of branched ester enolates and linear nitrile anions in intramolecular alkylations drew us into a study of the brefeldin problem. An additional reason was that it became a matter of honor (vide infra)!
A literature search revealed that Kitahara and Mori had employed the intramolecular nitrile anion alkylation (INAA) of nitrile 20 in their synthesis of brefeldin A, as depicted in Scheme 6. ${ }^{11}$ Given the presence of an acidic proton in product 21, one could argue whether or not the observed stereoselectivity $(92: 8)$ reflects a kinetic preference under the cyclization conditions (NaHMDS, benzene, reflux, $20 \mathrm{~min}$ ). Despite this concern, which could have jeopardized the logic behind our particular comparative study, we proceeded to examine the stereoselectivity of the intramolecular alkylation of ester counterpart 22 of the Mori nitrile, thinking this would take only several months. In reality, this issue occupied our attention for more than ten man years!
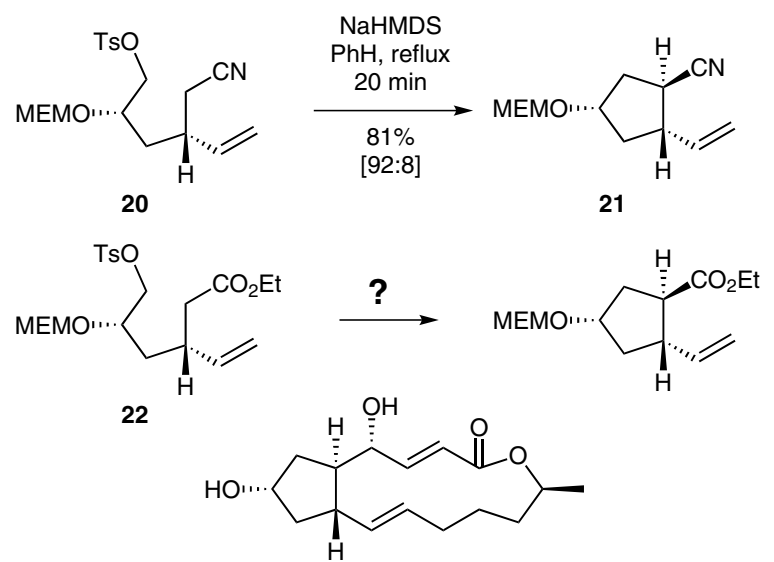

(+)-brefeldin A (19)

Scheme 6 Branched ester enolate versus linear nitrile anion alkylation

After considering synthetic routes to ester 22, we decided we might as well pursue the synthesis of a more advanced intermediate for brefeldin A, such as Bartlett intermediate 23. ${ }^{12}$ Shown in Scheme 7 is the initial retrosynthetic plan I conceived, somewhat naively in retrospect, and this was given to a part-time student who was doing his Ph.D. while working for a pharmaceutical company. It was patterned after the sequential acyclic Claisen rearrangement strategy used in Stork's ingenious prostaglandin synthesis. $^{13}$

The scheme seemed straightforward, except that the secondary hydroxy groups in diol $\mathbf{2 4}$ required differentiating for the sequential [3,3]-sigmatropic rearrangement. I believed that this could be achieved through the preferential intramolecular formation of a six-membered-ring mixed ketal in methanol, incorporating the homoallylic hydroxy group and thus exposing the allylic one, as depicted in compound 25 (Scheme 7).

My student, who was carrying out his research work at the company after work hours, made dihydroxy ketone $\mathbf{2 4}$ in no time and next attempted the intramolecular ketal formation in methanol as I suggested. He called me and said, "I don't get anything after column chromatography, although I saw a distinct nonpolar spot in the TLC of the re- 

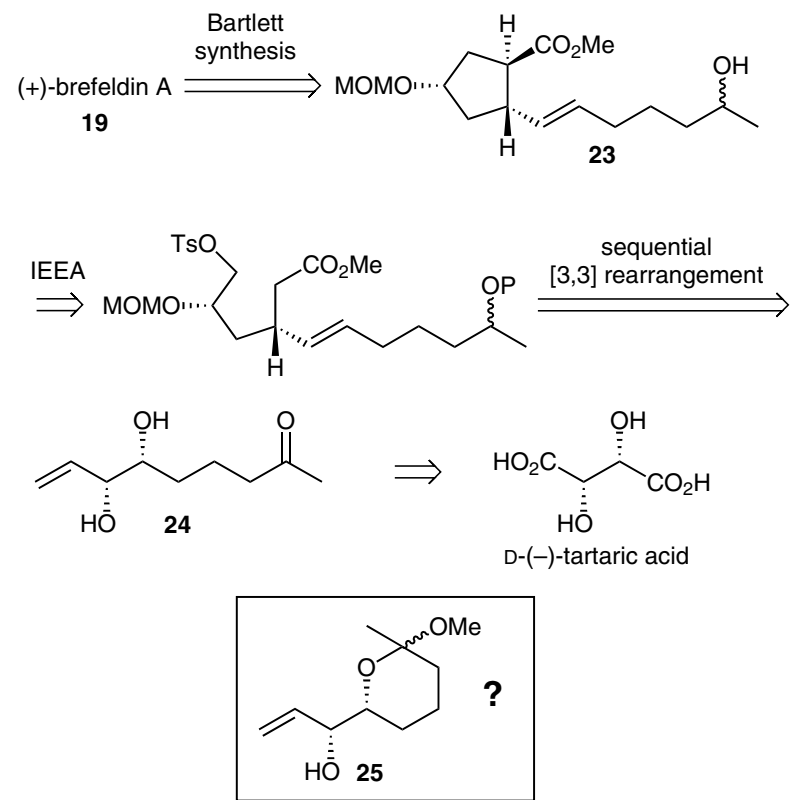

Scheme 7 Initial retrosynthetic plan for brefeldin A (19)

action mixture." That gave me an anti-eureka moment (!) because I had neglected the possibility of intramolecular bicyclic ketal formation occurring from the diol functionality in substrate $\mathbf{2 4}$ (Scheme 8) which nevertheless is well-documented in the synthesis of brevicomin. ${ }^{14}$ I told him to evaporate the eluent at a lower temperature and he brought me the NMR spectrum, which indicated we had accomplished an unintended formal synthesis of exobrevicomin (26).
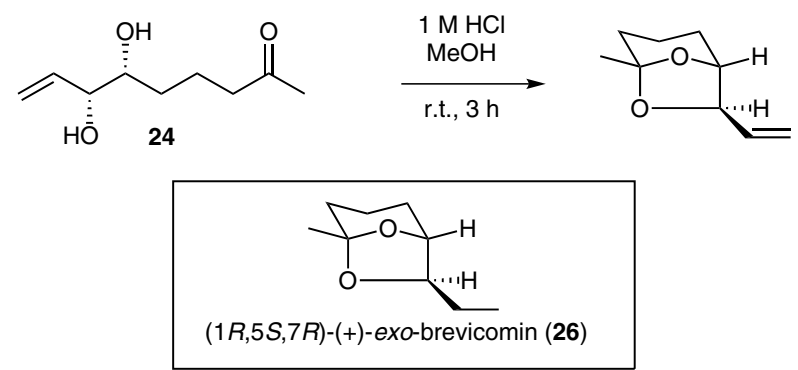

Scheme 8 Unintended formal synthesis of exo-brevicomin (26)

I had lost face, which is a grave matter to an East Asian professor, especially when my students thought I was so smart after they heard I was trained in a major research group in the United States! Desperate to save face by coming up with a superior synthesis of brefeldin A, I meticulously went over all the existing syntheses of this natural product, including total and formal syntheses and synthetic approaches - more than 20 of them at the time - during the whole summer vacation. ${ }^{10 \mathrm{~b}}$ At the time, brefeldin was a hot topic owing to its biological activity and interesting molecular structure. The list of researchers who investigated this problem read like a Who's Who of natural product synthesis chemists, and it was a tall order to come up with another and superior synthesis.
One day, out of the blue, an idea came to mind: why not let the chirality of the remote C-15 hydroxy group control the configuration of the rest of the stereogenic centers in the molecule by a triple chirality transfer process? Very fortunately, a synthetic plan fell into place like magic (Scheme 9). Thus, we accomplished a 12-step synthesis of the advanced brefeldin intermediate $\mathbf{2 8}$ from readily available ethyl $O$-benzyl lactate. Chemoselective sodium borohydride reduction of the $\alpha$-alkoxy ester in the third Claisen rearrangement product 27 in the presence of a simple ester was instrumental in its brevity. I still feel a bit odd claiming this as a 12-step synthesis while invoking a diazomethane workup twice as uncounted steps. Nevertheless, I consider this fortuitous synthesis one of the highlights of my entire career and a not inelegant recovery from an embarrassing misjudgment.

\section{Intramolecular $\mathrm{S}_{\mathrm{N}} 2^{\prime}$ Enolate Alkylation}

Meanwhile, we were intrigued by a possible alternative to the previously described way of controlling the relative stereochemistry of three stereogenic centers in a single operation, i.e. different from the elaborate doubly diastereodifferentiating IEEA protocol. We reasoned that a folding and allylic strain controlled intramolecular $\mathrm{S}_{\mathrm{N}} 2^{\prime}$ enolate alkylation might offer such an alternative. ${ }^{15}$ Years ago, when I told Gilbert Stork that I was going back to Korea, he asked me whether I would have an NMR spectroscopy machine, and further mentioned that I could study $\mathrm{S}_{\mathrm{N}} 2^{\prime}$ reactions even under relatively spartan conditions. Obviously, his encouragement had a significant influence on my pursuing this avenue of inquiry.

Our successful efforts along these lines are exemplified in our synthesis of elemol (29), as summarized in Scheme $10 .{ }^{16}$ In this work, we established the relative stereochemistry of all three stereogenic centers in the natural product in a single operation using a folding strain controlled intramolecular $\mathrm{S}_{\mathrm{N}} 2^{\prime}$ ester enolate alkylation $(\mathbf{3 0} \rightarrow \mathbf{3 1})$, which probably proceeds through chairlike double Heclipsed transition state geometry $\mathbf{C}$, as depicted in Scheme 10.

With the advent of the ring-closing metathesis (RCM) methodology, it was quite fulfilling to revisit the steroidal trans-hydrindane problem by employing an intramolecular $\mathrm{S}_{\mathrm{N}} 2^{\prime}$ amide enolate alkylation strategy $(\mathbf{3 4} \rightarrow \mathbf{3 5})$ and RCM. Thus, we achieved a concise, seven-step synthesis of trans-hydrindane 32 from commercially available 2acetylcyclopentanone (33), as illustrated in Scheme 11. ${ }^{17}$

In an effort to apply our internal $\mathrm{S}_{\mathrm{N}} 2^{\prime}$ strategy to alkaloid synthesis, intramolecular $\mathrm{S}_{\mathrm{N}} 2^{\prime}$ lactam enolate alkylation was carried out on substrate $(E)-37$, which has an $(E)$-allylic chloride pendant moiety. Exposure of $(E)-37$ to potassium hexamethyldisilazide provided the desired spirocyclic intermediate 38 in a 43:1 isomeric ratio, and this was converted into isonitramine (36) in racemic form (Scheme 12). ${ }^{18}$ As in the previously described grandisol 
synthesis, the observed stereoselectivity in the case of the cyclic enolate was excellent.

Encouraged by the success of the double allylic strain controlled intramolecular $\mathrm{S}_{\mathrm{N}} 2^{\prime}$ strategy, we formulated a synthetic plan for the asymmetric synthesis of (+)-isonitramine $[(+)-36]$ based on a triple allylic strain controlled version. In this example, the chirality of the 1-phenylethylamine moiety in substrate $(E)$-39 induced that of the spirocyclic quaternary carbon atom in the desired intermediate $\mathbf{4 0}$ in a 1,4-fashion with a selectivity of 6.2:1 (Scheme 13). ${ }^{19}$

During our synthetic study on (+)-isonitramine, we made the very interesting and fortuitous observation on one occasion of the formation of a tiny amount of compound $\mathbf{4 1}$ in addition to the previously mentioned $6.2: 1$ stereoisomeric mixture of six-membered-ring $\mathrm{S}_{\mathrm{N}} 2^{\prime}$ product 40 (Scheme 14). ${ }^{20}$ The spectral data suggested an eight-membered ring structure for this minor product, and very fortunately we were able to ascertain that the starting material used in that particular experiment was contaminated with a small amount (ca. 5\%) of the corresponding $Z$-isomer.

Subsequently, we independently synthesized the isomeric allylic chloride (Z)-39 and subjected it to comparable cyclization conditions. Eight-membered ring product 41 was formed in high yield as a single diastereomer (the spirocyclic carbon stereochemistry was undetermined), identical to that obtained previously (Scheme 14).

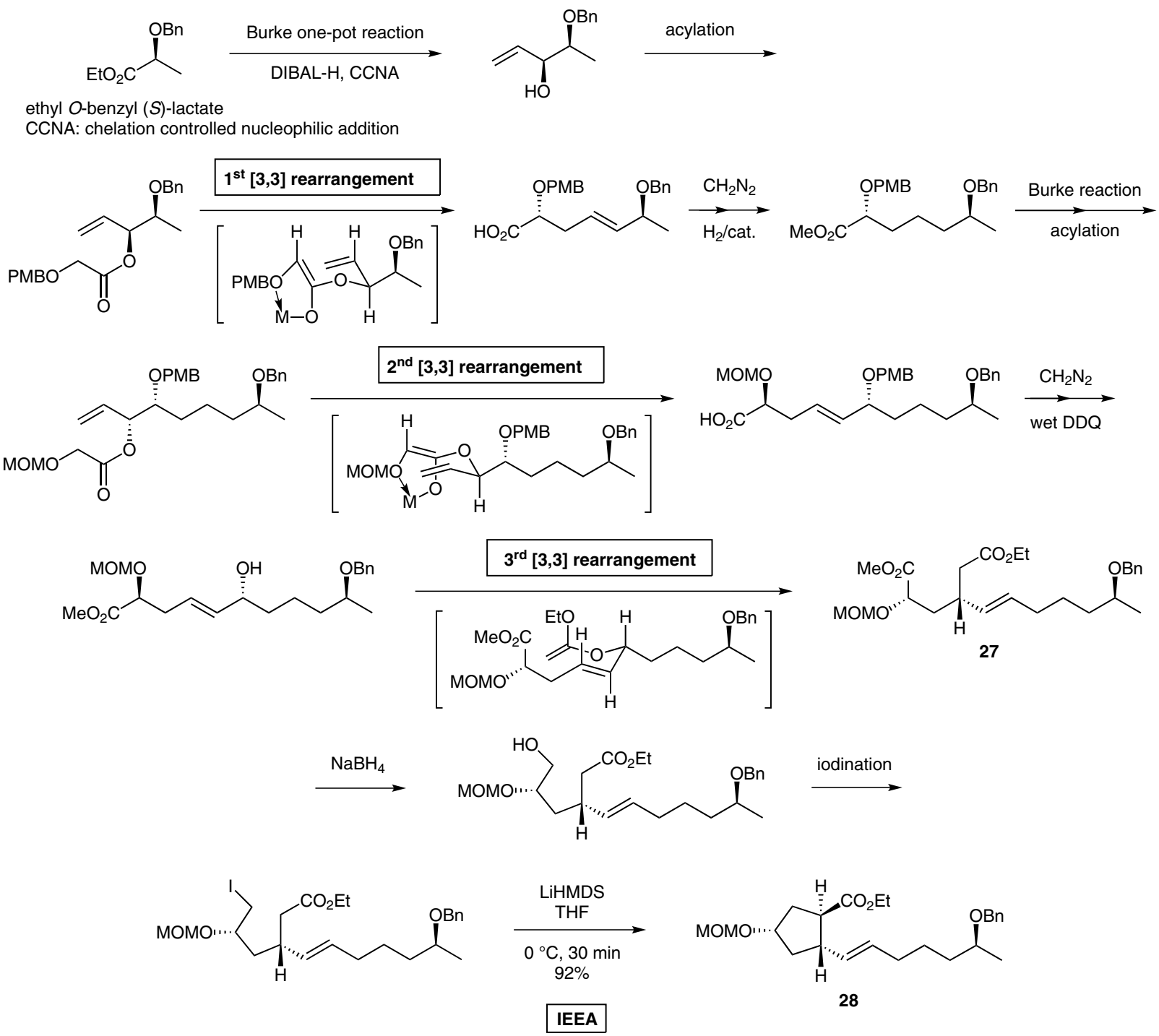

Scheme 9 Triple chirality transfer/intramolecular ester enolate alkylation strategy for brefeldin A (19) 


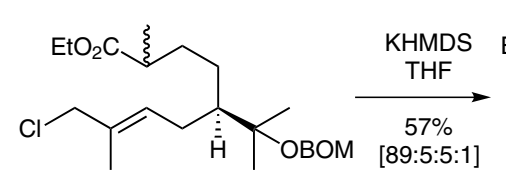

30

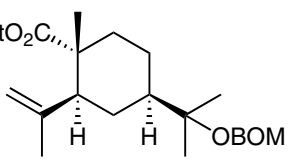

31
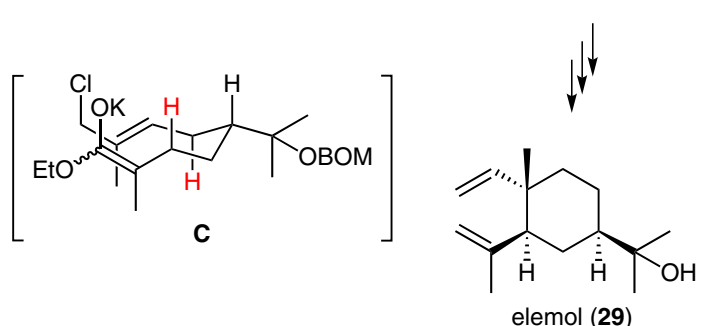

Scheme 10 Synthesis of elemol (29) via folding and double allylic strain controlled intramolecular $\mathrm{S}_{\mathrm{N}} 2^{\prime}$ ester enolate alkylation

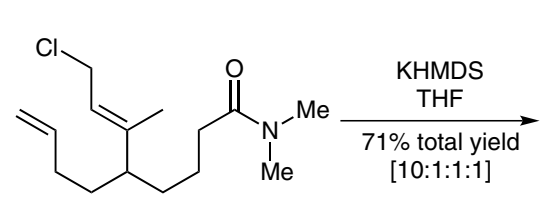

34<smiles>CC(=O)C1CCCC1=O</smiles>

commercially available<smiles>C=CCCC1CCC(C(=O)N(C)O)C1(C=C)C(=O)N(C)O</smiles>

35
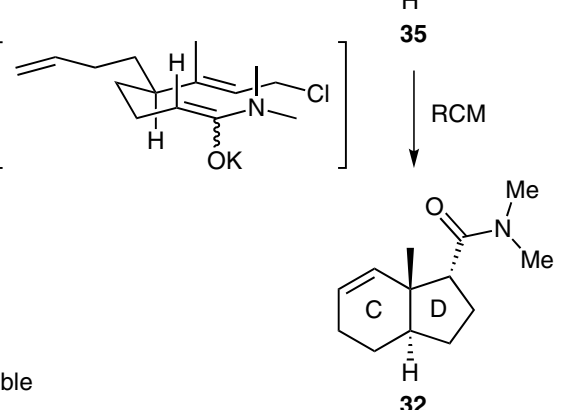

Scheme 11 Concise construction of steroidal trans-hydrindane 32 using a folding and allylic strain controlled intramolecular $\mathrm{S}_{\mathrm{N}} 2^{\prime}$ alkylation/ring-closing metathesis strategy

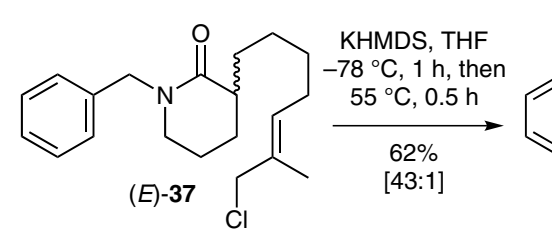

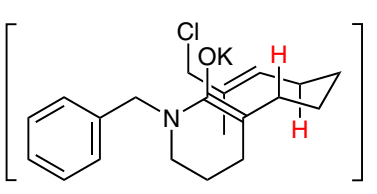<smiles>[Y][Y](C)=CC</smiles>

rac-isonitramine (rac-36)

Scheme 12 Synthesis of racemic spirocyclic alkaloid isonitramine (36) via double allylic strain controlled intramolecular $\mathrm{S}_{\mathrm{N}} 2^{\prime}$ lactam enolate alkylation

Next, we made a large quantity of the corresponding simpler $N$-benzyl compound (Z)-37 to investigate this inter-
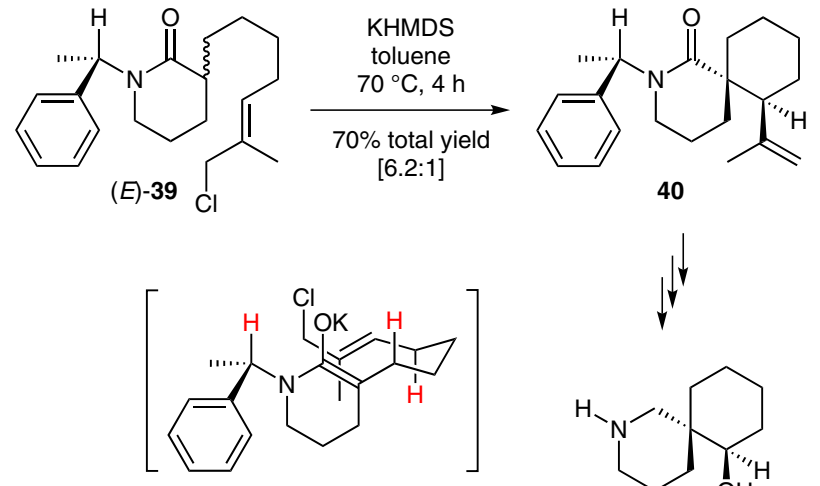

$\mid$

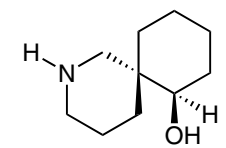

(+)-isonitramine $[(+)-36]$

Scheme 13 Asymmetric synthesis of (+)-isonitramine [(+)-36] via triple allylic strain controlled intramolecular $\mathrm{S}_{\mathrm{N}} 2^{\prime}$ lactam enolate alkylation

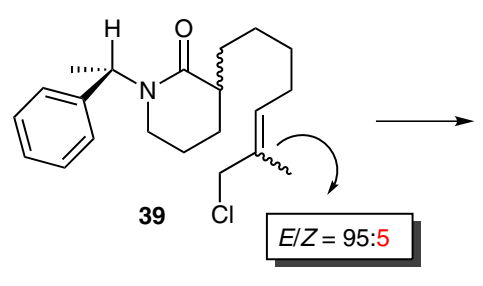<smiles>C=C(C)[C@H]1CCCC[C@]12CCCN([C@H](C)c1ccccc1)C2=O</smiles>

40

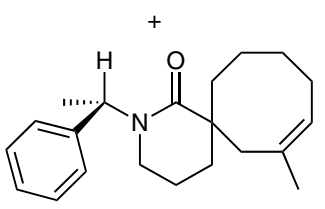

41

(a very small amount)<smiles>C/C1=C/CCCCC2(CCCN([C@H](C)c3ccccc3)C2=O)CC1</smiles>

(Z)-39<smiles>C/C1=C/CCCCC2(CCCN(Cc3ccccc3)C2=O)CC1</smiles>

$(Z)-37$<smiles>C/C1=C/CCCCC2(CCCN([C@H]([CH]c3ccccc3)c3ccccc3)C2=O)C1</smiles>

41 esting observation in detail. However, we were quite surprised to find that this $(Z)$-allylic chloride, which lacks a methyl group at the benzylic position, did not produce any of the desired product under comparable conditions (Scheme 14). This was probably due to the acidity of the benzylic hydrogens under the strong basic reaction conditions.

As far as we are concerned, this failure because of such a subtle difference is quite significant. Had we not tried the asymmetric version of the isonitramine synthesis, we might have never discovered the efficient methodology for the construction of medium-sized rings which I will describe from here on. 


\section{Synthesis of $\alpha, \alpha^{\prime}$-cis-Disubstituted Medium- Ring Oxacyclic Marine Natural Products}

Owing to my obtuse nature, as well as our obsession with the stereoselective creation of quaternary centers, we did not fully appreciate the synthetic potential of the above serendipitous discovery for quite some time. It was not until medium-ring oxacyclic marine natural products began to succumb to total synthesis with the advent of the RCM methodology, notably achieved by the research group of Professor Michael Crimmins, that we turned our attention to this area. We envisaged that the adaptation of our fortuitous finding to the key step of intramolecular glycolate enolate alkylation, which we employed previously in our fumagillol synthesis (see Section 2), might offer us a viable synthetic strategy for the construction of medium-ring oxacycles.

Our model study to test the feasibility of this approach led to what we term the 'olefin geometry dependent internal alkylation' strategy, where the geometry of the olefin controls the mode of cyclization, as illustrated in Scheme $15{ }^{21}$ Thus, trans-olefin $(E)-\mathbf{4 2}$ gave rise predominantly to six-membered ring 43 through an $\mathrm{S}_{\mathrm{N}} 2^{\prime}$ pathway as anticipated, while cis-olefin (Z)-42 produced mainly eightmembered ring 44 via a simple $\mathrm{S}_{\mathrm{N}} 2$ reaction.<smiles>CN(C)C(=O)COCCCC=CCCl</smiles>

$(E)-42$<smiles>CN(C)C(=O)COCCC/C=C/CCl</smiles>

(Z)-42
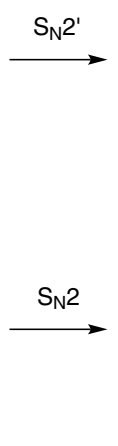

Scheme 15 Olefin geometry dependent intramolecular amide enolate alkylation

With this encouraging result, the focus of our research shifted to the asymmetric total synthesis of medium-ring oxacyclic marine natural products. Figure 1 illustrates the structures of those natural products whose syntheses are covered in this account. Some of the medium-ring oxacyclic marine natural products possess so-called $\alpha, \alpha^{\prime}$-cis side chain appendages and others have the $\alpha, \alpha^{\prime}$-trans arrangement. I have used the colors violet and green to indicate the cis and trans relationships, respectively, throughout the rest of this account. In addition, I would like to point out that both $Z$ - and $E$-isomers are found in nature in the case of oxonenes.

Before further discussing our work, I would like to mention that we have pursued efficient, 'entirely substratecontrolled' asymmetric total synthesis wherever appropri-

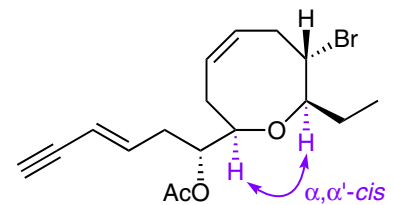

(+)-laurencin

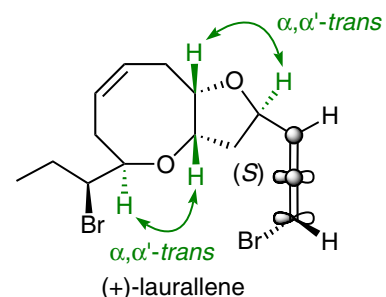

(+)-laurallene

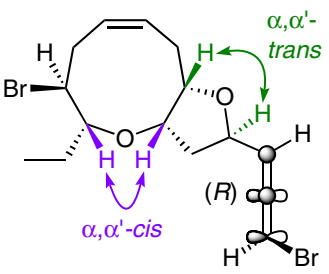

(-)-isolaurallene

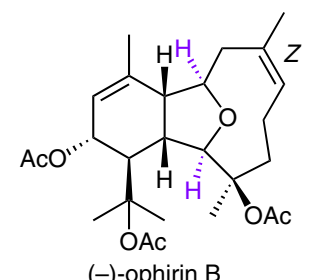

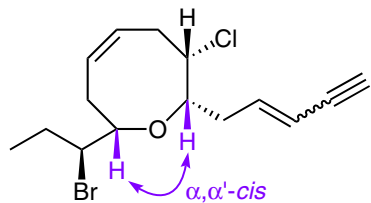

$(+)-(3 E)-$ and $(+)-(3 Z)-$ pinnatifidenyne

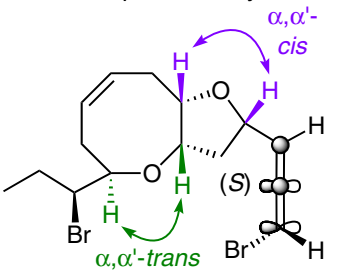

(+)-pannosallene

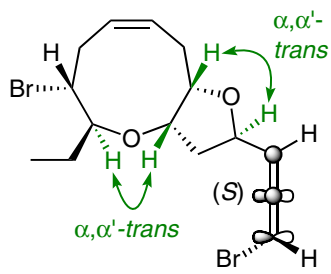

(+)-itomanallene A

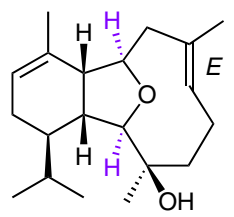

(-)-cladiella-6,11-dien-3-ol
Figure 1 Representative medium-ring oxacyclic marine natural products

ate. In connection with this, Smith and Empfield introduced the concept of a 'stereochemically linear strategy' for this approach. ${ }^{22}$ To track this strategy more easily, I have used a black asterisk circled in yellow to indicate the origin or source of absolute chirality, and red and blue asterisks to denote stereocenters induced by substrate control and asymmetric methods, respectively. In addition, the size of each asterisk indicates the sequence in which the corresponding stereocenter was introduced; the larger the asterisk, the later it was introduced. This notation, which is used throughout the rest of this account, is illustrated in Scheme 16 using the previously described synthesis of (+)-brefeldin A as an example (see Section 2).

The problems we needed to address for synthesis of the medium-ring oxacyclic natural products were as follows: Firstly, one must construct the medium-ring oxacyclic skeleton while dealing with the very important problem of controlling the relative stereochemistry of the two side chain appendages, which are either $\alpha, \alpha^{\prime}$-cis or $\alpha, \alpha^{\prime}$-trans. Secondly, one must then deal with the elaboration of the flexible side chain, which often possesses stereogenic centers, enynes, bromoallene moieties, etc. Finally comes the introduction of a halogen atom with a defined stereochemistry, which in my opinion is the most demanding task. It turns out that this seemingly simple synthetic op- 


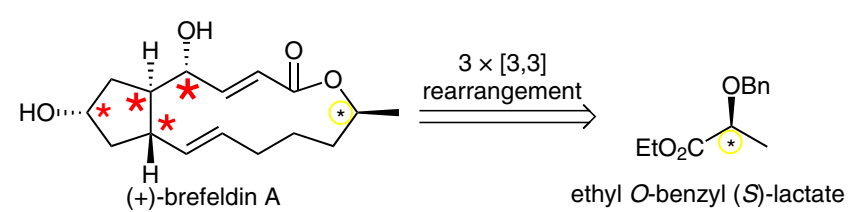

* : source of absolute chirality

* : by substrate control

* : by asymmetric methods

the larger the asterisk, the later it was introduced

Scheme 16 Substrate-controlled asymmetric synthesis and a key to the notations used in this account

eration often dictates the course of the entire synthesis ( $v i$ de infra).

The next portion of this account is a series of discussions on our approaches to each of the target compounds depicted in Figure 1, with a focus on the crucial synthetic transformations. Keeping in mind our self-imposed stereochemical goal and synthetic tasks, first I will discuss our substrate-controlled synthesis of $\alpha, \alpha^{\prime}$-cis-natural products. I will begin with a detailed examination of our synthesis of (+)-laurencin, the most well-known metabolite of this class, to give you an idea of the type of objectives we were pursuing. ${ }^{23}$

With the above in mind, the retrosynthetic plan for our $(+)$-laurencin synthesis, depicted in Scheme 17, was quite straightforward. It involved the preparation of the internal alkylation substrate $(\mathbf{4 9} \rightarrow \mathbf{4 8})$, its intramolecular amide enolate alkylation (IAEA) $(\mathbf{4 8} \rightarrow \mathbf{4 7})$, elaboration of the side chain appendage $(\mathbf{4 7} \rightarrow \mathbf{4 6})$, and bromination $(46 \rightarrow$ 45), this latter step already being known.

The preparation of the key internal alkylation substrate $\mathbf{4 8}$ is shown in Scheme 18. Thus, an Evans-Crimmins asymmetric alkylation $(\mathbf{4 9} \rightarrow \mathbf{5 0})^{24}$ and Burke's one-pot reduction/chelation-controlled nucleophilic addition protocol

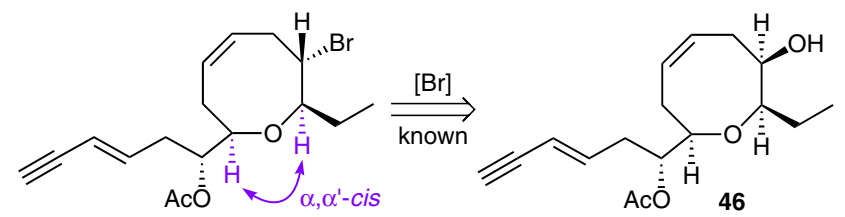

(+)-laurencin (45)

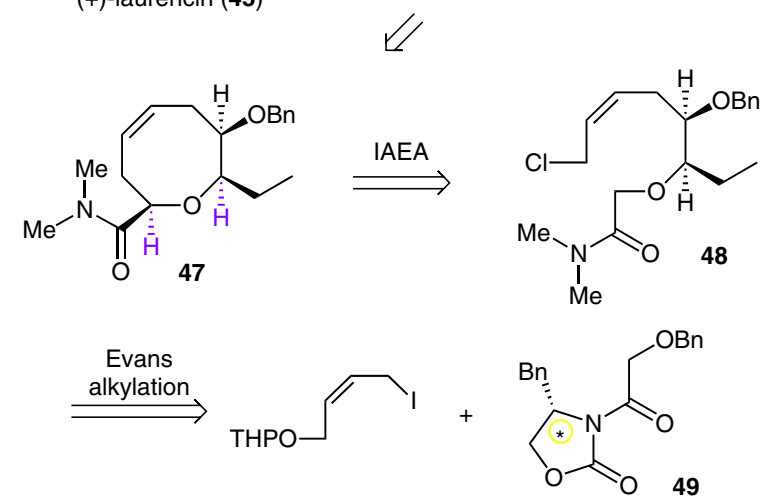

Scheme 17 Retrosynthetic plan for (+)-laurencin (45) $(\mathbf{5 1} \rightarrow \mathbf{5 2})^{25}$ were utilized to establish the target compound's $\mathrm{C}-12 / \mathrm{C}-13$ absolute and vicinal relative stereochemistry. A direct, three-step sequence then afforded the requisite internal alkylation substrate $\mathbf{4 8}$ in reasonable overall yield (38\% for the six steps from 49$)$.
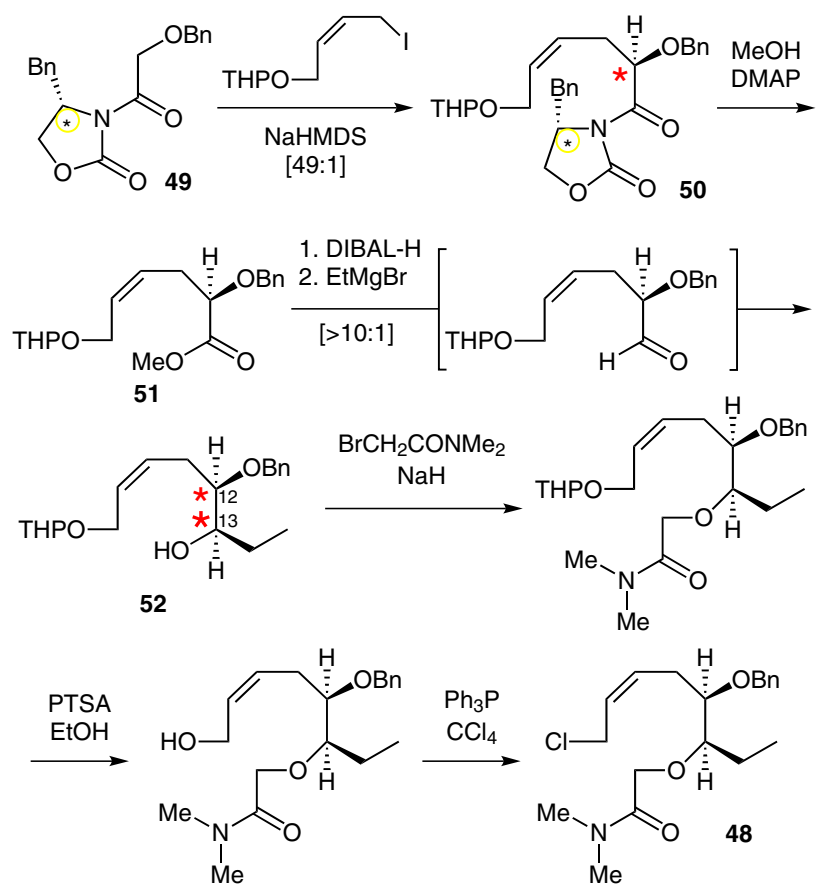

Scheme 18 Preparation of internal alkylation substrate 48

We were very pleased to find that the application of our IAEA to ( $Z$ )-allylic chloride $\mathbf{4 8}$ proceeded quite smoothly to give the desired $\alpha, \alpha^{\prime}-c i s$-oxocene $\mathbf{4 7}$ in excellent yield (Scheme 19). The $\alpha, \alpha^{\prime}-$ cis relative stereochemistry of product $\mathbf{4 7}$ was firmly established using nuclear Overhauser effect (NOE) spectroscopic studies (Scheme 19). This high degree of regioselectivity was gratifying, although it is hard to craft a convincing rationale as to why oxocene 47 was formed at the expense of the $\mathrm{S}_{\mathrm{N}} 2$ ' product via a six-membered-ring transition state. However, given the potential utility of this transformation, we plan further investigations into the underlying reasons for the observed regioselectivity $\left(S_{N} 2\right.$ versus the $S_{N} 2^{\prime}$ pathway) in this IAEA reaction.

It is worth mentioning that our plan hinged upon the versatility of the $\alpha$-alkoxy-substituted $N, N$-dimethylamide functional group as illustrated with oxocene targets in Scheme 20 . We had elected to use a $N, N$-dimethylamide group for the internal alkylation simply because the corresponding ester was unsatisfactory, and at that time we did not fully appreciate what its value would be.

The addition of organometallics, such as Grignard or alkyllithium reagents, to the $\alpha$-alkoxy-substituted $N, N$-dimethylamide gave the corresponding ketone directly in a highly reliable manner (Scheme 20). I imagine that the subconscious inspiration for this process came from the Weinreb ketone synthesis ${ }^{26}$ which proceeds via a very 


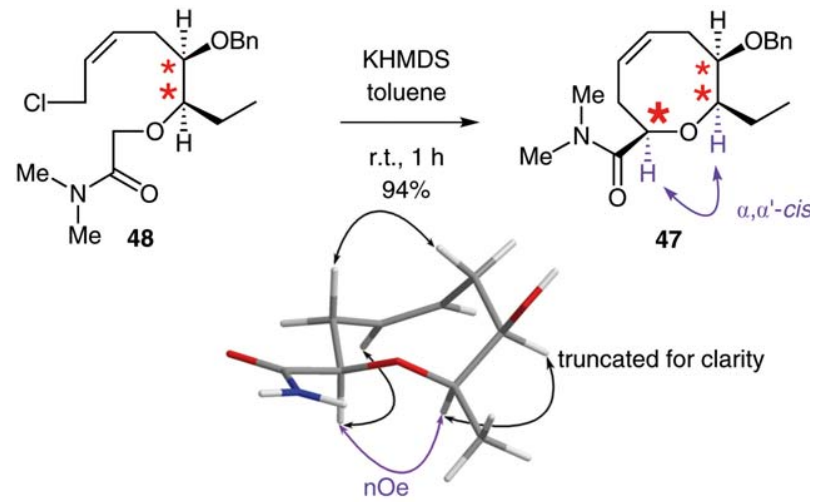

Scheme 19 Intramolecular amide enolate alkylation

stable metal-chelated intermediate like that shown for the above direct ketone synthesis protocol. L-Selectride reduction of the resultant ketone is known to proceed in a highly stereoselective fashion, rationalized by FelkinAhn model D. ${ }^{27}$

The existing syntheses in this area usually employ a rather cumbersome sequence, i.e. the non-stereoselective addition of an organometallic species to an aldehyde, separation of the mixture, then reoxidation and L-Selectride reduction. Our versatile three-step sequence, i.e. IAEA, direct ketone synthesis, and L-Selectride reduction, constitutes the backbone of a synthetic strategy that enabled us to achieve concise and efficient syntheses of our target compounds, our routes being competitive with or surpassing existing approaches in terms of brevity. In addition, the $N, N$-dimethylamide could be converted into the corresponding aldehyde or primary alcohol by addition of an ate complex or Superhydride, respectively (Scheme 20).

As I pointed out earlier, the introduction of the halogen atom in these target compounds in a stereochemically controlled manner is a considerable challenge. Figure 2 summarizes our rather extensive experience ${ }^{28 a}$ of the halogenation of the four possible diastereomeric oxocenyl alcohols with inversion of configuration under Hooz's conditions. ${ }^{28 b}$ Whatever the exact reason might be, the ha- logenation of the $\alpha, \alpha^{\prime}$-cis-syn-oxocenyl alcohol (syn with respect to $\mathrm{C}-12 / \mathrm{C}-13$ in laurencin) usually gives a satisfactory result. Halogenation of the cis-anti- and trans-synisomers tends to be problematic. However, the trans-antiisomer represents the worst substrate for halogenation. Throughout this account, I will be using the 'ease-of-halogenation' rating system shown in Figure 2, where the valence and magnitude are indicated by the plus and minus signs in the parentheses.
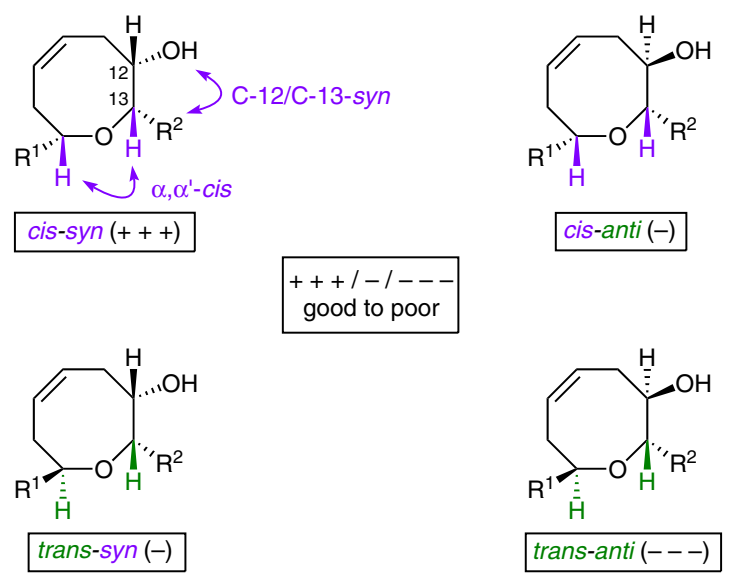

Figure 2 Ease of the halogenation of diastereomeric oxocenyl alcohols (involving inversion of configuration)

To complete the synthesis of (+)-laurencin (45), the acetonitrile anion was used as an acetaldehyde equivalent for a direct ketone synthesis $(\mathbf{4 7} \rightarrow \mathbf{5 3})$ (Scheme 21$)$. Stereoand chemoselective L-Selectride reduction $(\mathbf{5 3} \rightarrow \mathbf{5 4})$, followed by conventional steps including a stereoselective Wittig reaction $(\mathbf{5 5} \rightarrow \mathbf{5 6})$, led to the final product.

In summary, an efficient 15-step total synthesis of (+)-laurencin (45) was accomplished in a completely substratecontrolled fashion, starting from known glycolate derivative 49 as the source of absolute chirality (Scheme 22).

Would you believe it if I said that Steven Weinreb edited ALL my manuscripts for more than two decades saying

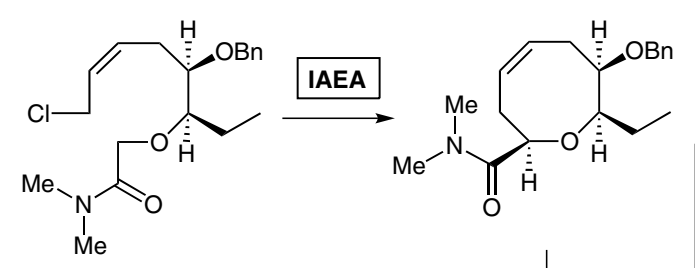<smiles>CN(C)C1(P)CON(C2CC3CCC2C3)O1</smiles>

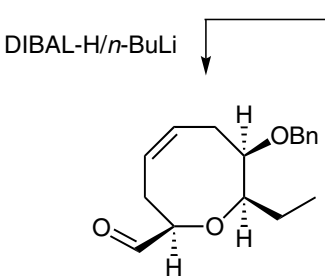

$\mathrm{RM}=$ organometallic reagent
Superhydride<smiles>CCC1O[C@@H](CO)CC=CC[C@H]1OCc1ccccc1</smiles><smiles>[R]C(=O)C1CC=CC[C@H](O)C1CC</smiles><smiles>CC[C@H]1O[C@H](C(O)Cc2ccccc2)CC=CC[C@@H]1C(=O)P</smiles>

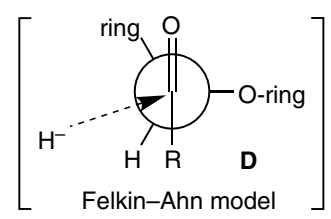

Felkin-Ahn model

Scheme 20 Versatility of the $\alpha$-alkoxy-substituted $N, N$-dimethylamide functional group 


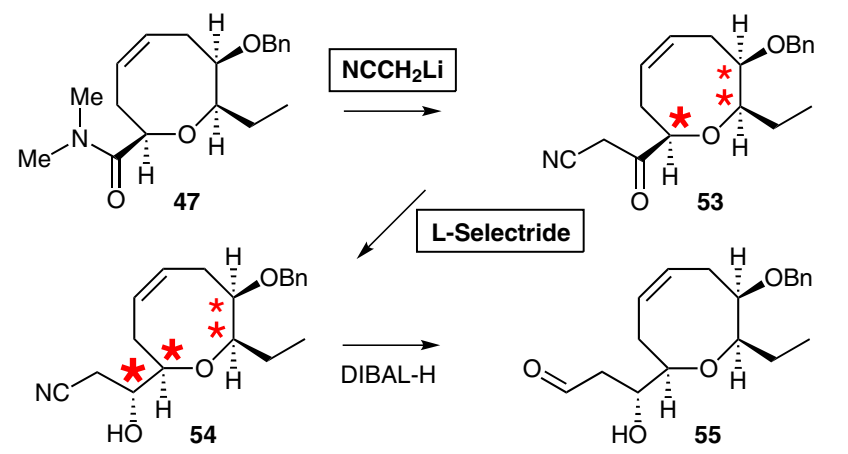<smiles>CCO[C@H]1CC=CCC([C@H](O)C/C=C/C#CS)OC1CC</smiles>

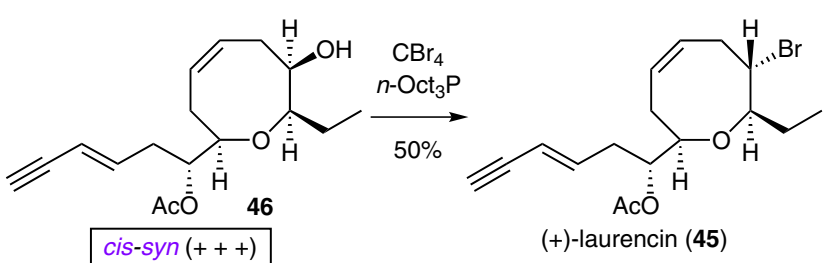

Scheme 21 Completion of the synthesis of (+)-laurencin (45)

that he wanted to know what I was doing in Korea. Lucky for him that I was not more productive! In the olden days, he would return the edited manuscript marked up by hand in a red pen via air mail. Oddly enough, I have never visited him at Penn State University, but I can easily imagine him doing the 'Weinreb Walk' to the post office with a can of Diet Pepsi in hand.

Recently, I decided to take the load off him considering his advancing age, and more importantly coinciding with the departure of one of his Ph.D. students, my former master's student, who had acted as a liaison in this new age of electronic communication. I do not know how else I can repay Steve's boundless and enthusiastic efforts as my mentor except by wishing him the very best of luck and health, and by taking the opportunity in writing this account to acknowledge the essential beneficial effect he has had on my career.

I imagine that chemists who have read my very recent papers must have noticed that my English shows sudden tremendous improvement. I know there are rumors floating around to the effect that "Kim must have a live-in Englishspeaking paramour". But the truth is that destiny brought me back together again with a labmate at Columbia, Dr. Matthew Schlecht, who is now doing chemistry with words in a practice named Word Alchemy. My cybermate and I exchange many, many rounds of editing and discussion for a manuscript - incidentally, he did his doctoral research on enolate chemistry, so it was a natural fit.

From key IAEA product $\mathbf{4 7}$ in the laurencin synthesis, we have developed a highly efficient, biomimetic route to (-)-laurefucin (57) via an organoselenium-mediated oxonium ion formation/fragmentation protocol $(\mathbf{5 8} \rightarrow \mathbf{5 9})$, as outlined briefly in Scheme 23..$^{29}$ As you might have guessed by now, as with much of my work, this novel organoselenium-based methodology benefited from an element of serendipity.

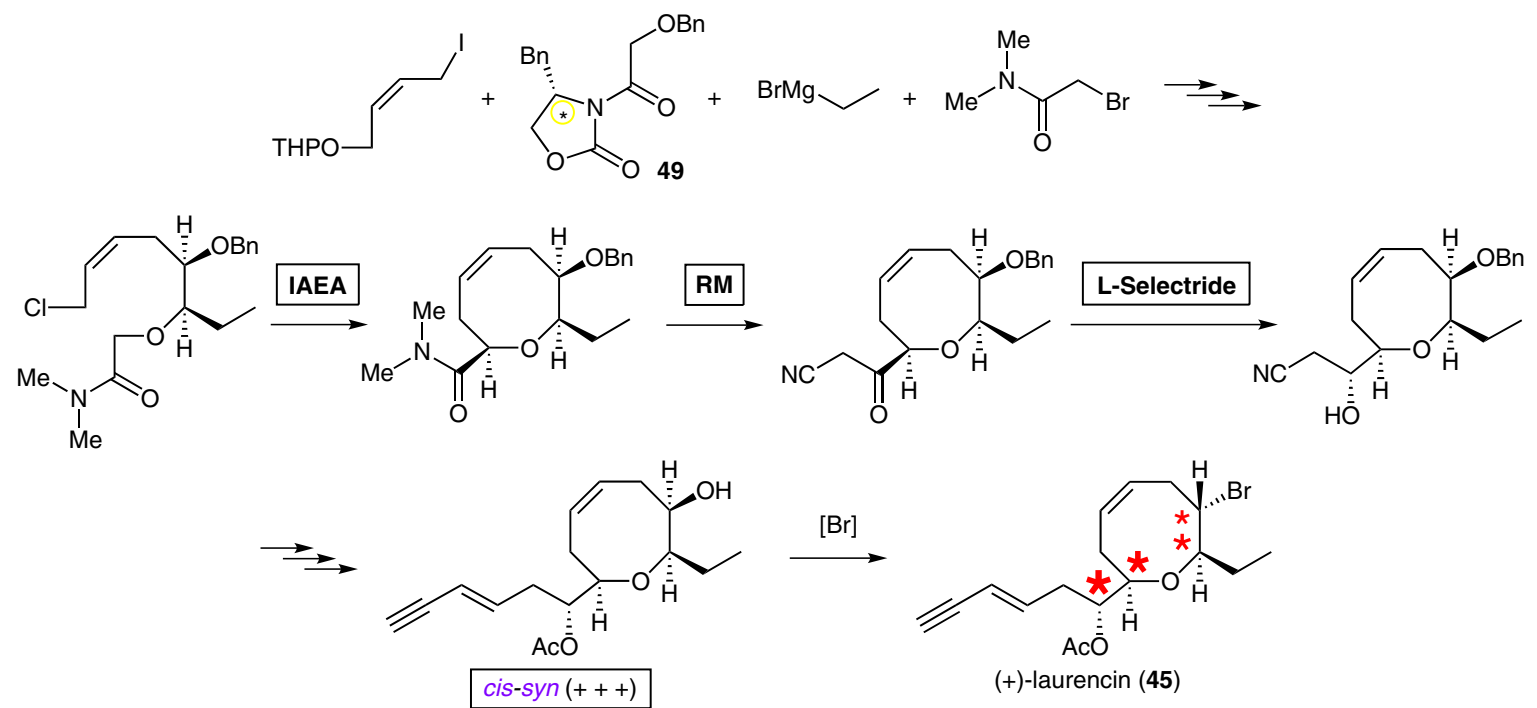

Scheme 22 Summary of the synthesis of (+)-laurencin (45) 
Taking advantage of the known natural product chemistry in the field, we further modified the organoseleniumbased methodology to arrive at a one-pot tandem organoselenium-mediated oxonium ion formation/silica gel promoted fragmentation protocol. This was used to achieve the asymmetric total synthesis of Laurencia natural products that have a $2,2^{\prime}$-bi(tetrahydrofuranyl) skeleton. ${ }^{28 a}$

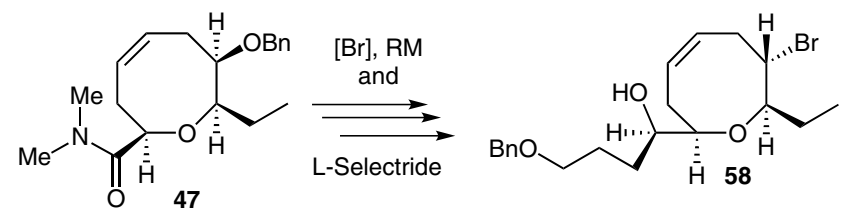

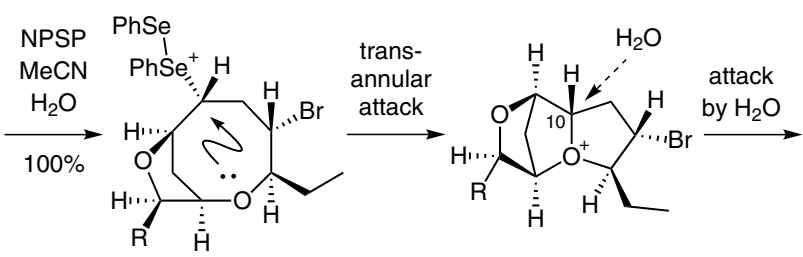

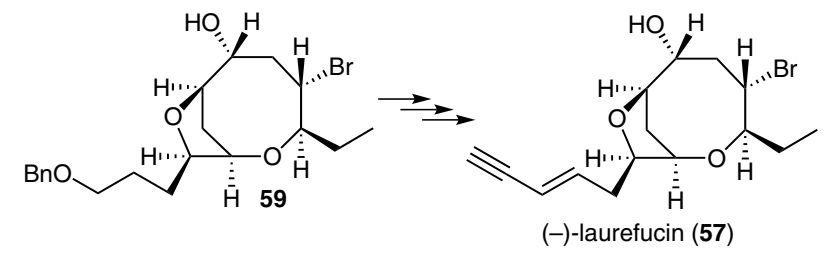

Scheme 23 Organoselenium-mediated hydroxyetherification route to $(-)$-laurefucin (57)

We found it intellectually amusing that we parlayed the biogenesis-inspired strategy for $\mathrm{C}_{15}$ Laurencia acetogenins into an asymmetric total synthesis of trilobacin (60) (Scheme 24), an annonaceous acetogenin of a completely different biogenetic origin which possesses potent anticancer activity. ${ }^{30}$ It is noteworthy that an entirely substrate-controlled approach for this molecule did not seem to be appropriate; the blue asterisks simply twinkle in the distance like unreachable stars, unable to communicate with the stereochemical core.
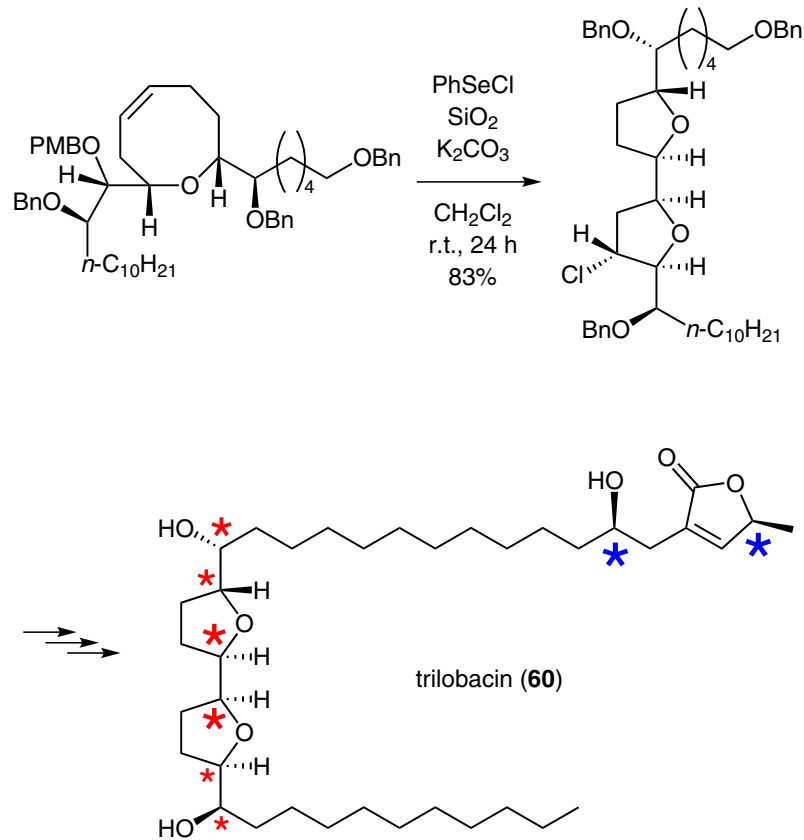

Scheme 24 Asymmetric total synthesis of trilobacin (60) via one-pot tandem organoselenium-mediated oxonium ion formation/silica gel promoted fragmentation

We addressed an internal alkylation issue in our synthesis of (+)-(3Z)-pinnatifidenyne (61), as depicted in Scheme 25 . The course of the synthesis was determined by the seemingly insignificant difference between our target compound 61 and (+)-laurencin (45), i.e. the relative stereochemistry of the ring halogen atom with respect to the adjacent alkyl group. ${ }^{21}$ The chlorination of cis-anti-oxocenyl alcohol 62 derived using our internal alkylation was problematic owing to a competing elimination reaction, and it produced only a modest yield $(33 \%)$ of the desired oxocenyl chloride 63.

To overcome this obstacle, we were forced to explore changing the order of events. Although it seemed rather risky at the outset, we were delighted to find that the acyclic chlorination/internal alkylation sequence proved to be highly efficient and afforded the desired oxocene $\mathbf{6 3}$ in good overall yield, demonstrating the synthetic potential of our methodology. 


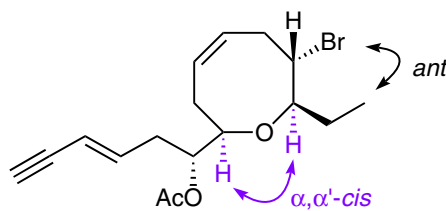

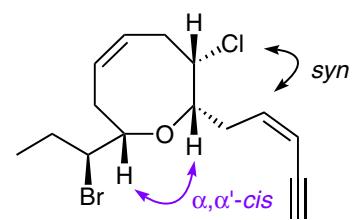

(+)-laurencin (45)

(+)-(3Z)-pinnatifidenyne (61)

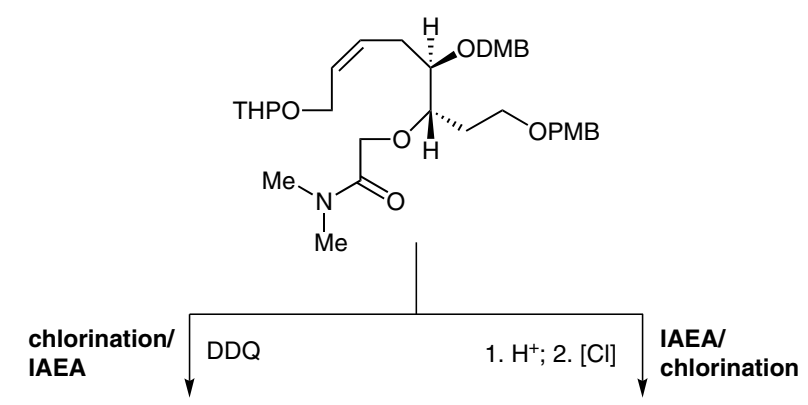

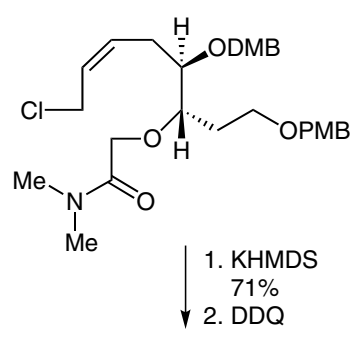

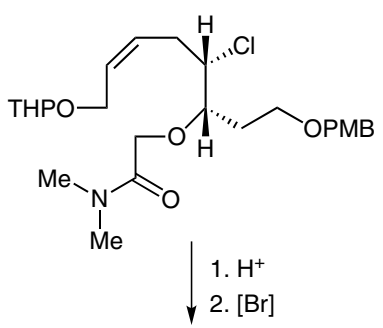

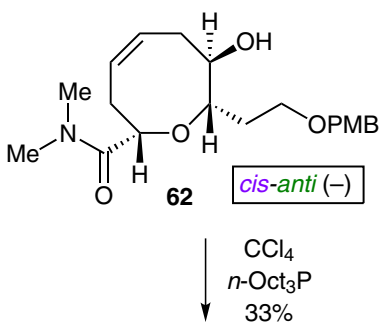<smiles>COCC[C@H](OCC(=O)N(C)C)[C@H](Cl)C/C=C\CBr</smiles>

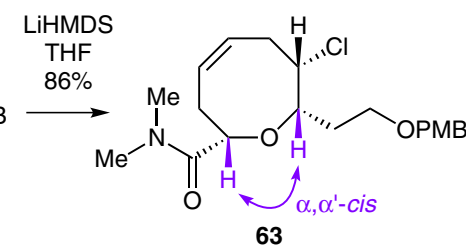

Scheme 25 Highlights in the asymmetric total synthesis of (+)-(3Z)pinnatifidenyne (61)

As the final subject of our synthesis of $\alpha, \alpha^{\prime}$-cis-disubstituted natural products, I will describe our endeavors toward the asymmetric total synthesis of cladiellin diterpenes, which exhibit a wide range of biological activities. ${ }^{31}$ As I mentioned earlier, both $Z$ - and $E$-isomers of these compounds are found in nature. These complex natural products possess an $\alpha, \alpha^{\prime}$-cis-disubstituted nine-membered ether ring and a tertiary carbinol moiety at C-3, as depicted in Scheme 26. The relative stereochemistry of the C-2 and C-3 oxygen functionalities is syn.

The Crimmins group reported the elegant syntheses of (Z)-cladiellin diterpenes, such as (-)-ophirin B (64) (Scheme 26), which employed a key RCM/intramolecular Diels-Alder (IMDA) strategy. ${ }^{32}$ We developed a general strategy for both (Z)- and (E)-cladiellins based on an IAEA/IMDA protocol, and in this account I will focus on the synthesis of $(E)$-cladiellin diterpenes, e.g. (-)-cladiella6,11-dien-3-ol (65), for which we believe our approaches have a distinct edge. ${ }^{33}$

As shown in Scheme 26, (Z)-C-3 tertiary $\left(3^{\circ}\right) / \alpha, \alpha^{\prime}$-cissyn-oxonene ( $Z$ )-67 could be constructed efficiently by applying our IAEA to $(Z)$-allylic chloride $(Z)$-66. Incidentally, the product of the Superhydride reduction of $(Z)$-oxonene ( $Z$ )-67 is a known Crimmins intermediate for (-)ophirin B (64). ${ }^{32}$ In the case of the $E$-isomer $(E)-66$, however, the chemical yield for the IAEA was low and capricious.

After some experimentation, we opted to remove the methyl group at $\mathrm{C}-3$ on the premise that the requisite tertiary carbinol moiety in the natural product could be created in the last stages of the synthesis based on Professor Leo Paquette's important finding in the $Z$ series. ${ }^{34}$ Gratifyingly, the internal alkylation of secondary derivative $(E)-\mathbf{6 8}$ was superior to that of its tertiary syn-counterpart (E)-66 in terms of yield and reproducibility.

The presence of the Kishi free radical inhibitor butylated hydroxytoluene (BHT) was critical for the success of the intramolecular Diels-Alder reaction of $(E)$-oxonene $\mathbf{7 0}$, derived from key IAEA product $(E)-69$; the reaction delivered a good yield of the desired adduct 71 (Scheme 27). The conversion of tricyclic intermediate 71 into our target molecule, (-)-cladiella-6,11-dien-3-ol (65), constituted the first total synthesis of an (E)-cladiellin (Scheme 27). One of the requirements of this total synthesis was to transform the ester functionality in intermediate $\mathbf{7 1}$ into an isopropyl group. This was achieved by reductive removal of the corresponding tertiary acetate with metallic potassium using the Barton protocol $(\mathbf{7 2} \rightarrow \mathbf{7 3}) .{ }^{35}$

Our synthesis of the $(E)$-cladiellin diterpene remained the first and only example until the very recent elegant work of Clark and co-workers. ${ }^{36}$ I confess that I had prayed every day for no one else to develop a synthesis of an $(E)$ cladiellin that would overshadow our work, but apparently the prayer went unanswered.

Furthermore, we demonstrated that our synthetic $(E)$-cladiellin 65 could be transformed into other cladiellin members, such as (-)-cladiell-11-ene-3,6,7-triol (74), (-)-deacetoxyalcyonin acetate (75), and (+)-polyanthellin A (76). Scheme 28 summarizes these highly stereo-, regio-, and chemoselective approaches, which take advantage of the reactivity and conformational rigidity of the $(E)$-oxonene moiety in the oxatricyclic skeleton of compound $\mathbf{6 5}$. 


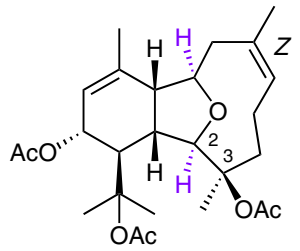

(-)-ophirin B (64)<smiles>CC(C)=CCC[C@](C)(Cc1ccccc1)[C@H](COCc1ccccc1)OCC(=O)N(C)C</smiles>

(Z)-66: (Z)-C-3 3\% syn

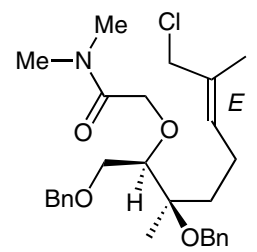

(E)-66: (E)-C-3 3\%/syn

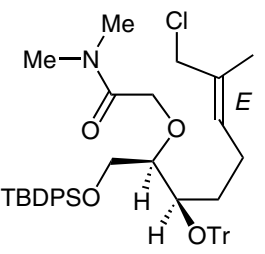

(E)-68: (E)-C-3 2\%syn

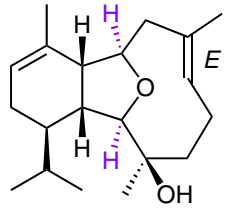

(-)-cladiella-6,11-dien-3-ol (65)

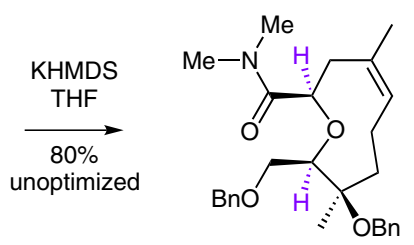

(Z)-67: (Z)-C-3 3\% $/ \alpha, \alpha^{\prime}-$ cis-syn

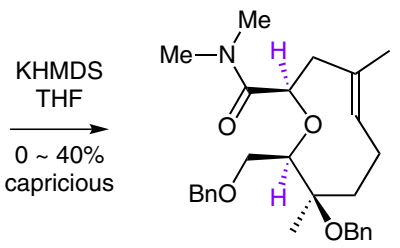

(E)-67: (E)-C-3 3\% $/ \alpha, \alpha^{\prime}$-cis-syn

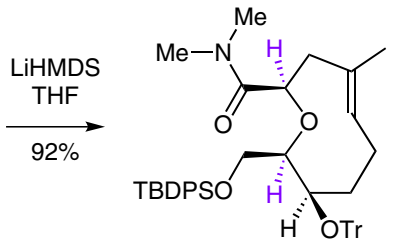

(E)-69: (E)-C-3 2\% $/ \alpha, \alpha^{\prime}-$ cis-syn
Scheme 26 Construction of both $(Z)$ - and $(E)$-oxonenes by intramolecular amide enolate alkylation
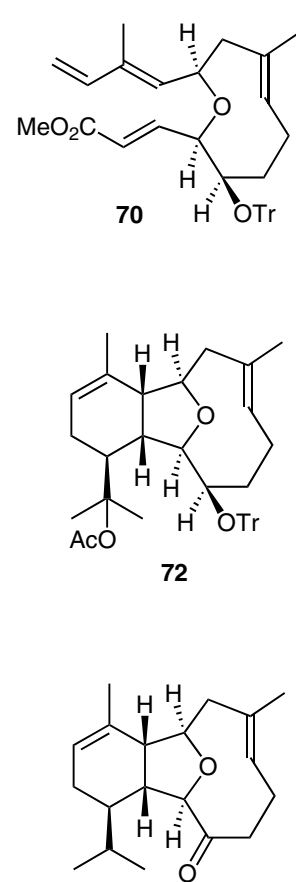
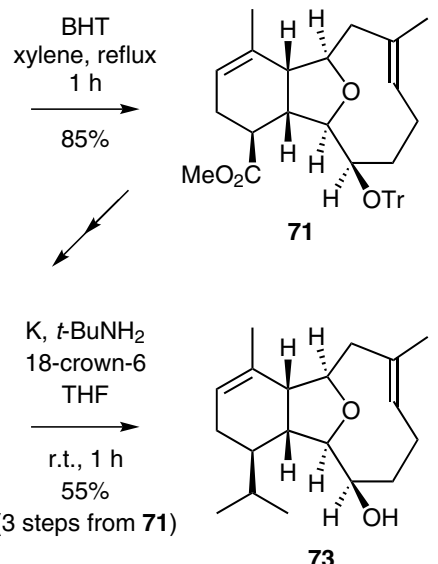

73
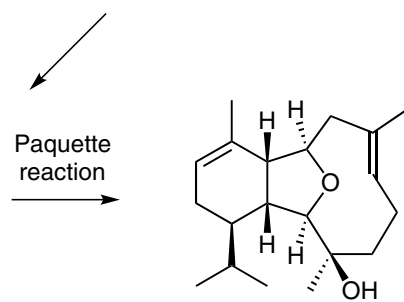

(-)-cladiella-6,11-dien-3-ol (65)
Scheme 27 Completion of the synthesis of (-)-cladiella-6,11-dien3-ol (65)

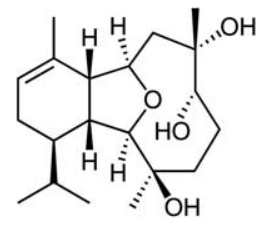

(-)-cladiell-11-ene-3,6,7-triol (74)

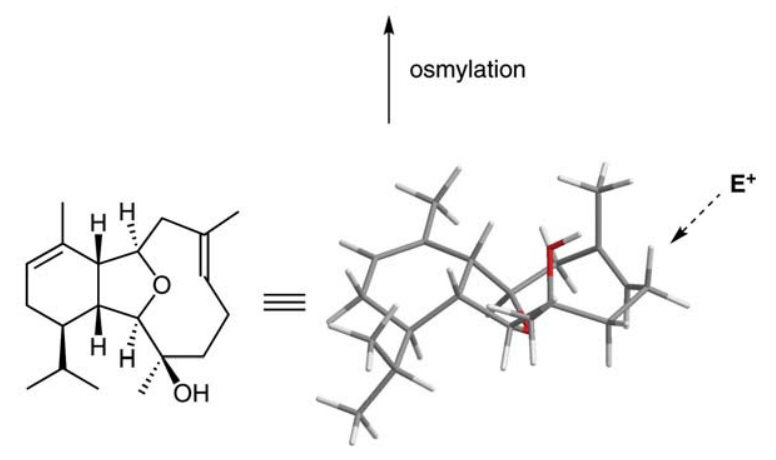

(-)-cladiella-6,11-dien-3-ol (65)

oxymercuration

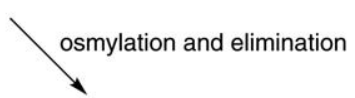

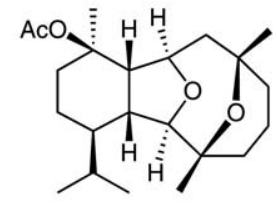

(+)-polyanthellin A (76)

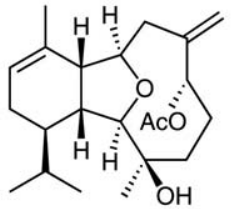

(-)-deacetoxyalcyonin acetate (75)
Scheme 28 Transformation of $(E)$-cladiellin 65 into other cladiellins

5

\section{Synthesis of $\alpha, \alpha^{\prime}$-trans-Disubstituted Medi- um-Ring Oxacyclic Marine Natural Products}

I hope the above discussion has demonstrated that our IAEA methodology has substantial potential for achieving substrate-controlled syntheses of $\alpha, \alpha^{\prime}$-cis-disubstituted medium-ring oxacyclic natural products.

For the remainder of this account, I would like to describe our efforts directed toward the synthesis of $\alpha, \alpha^{\prime}$-trans-disubstituted medium-ring oxacyclic natural products. Our IAEA methodology has not so far been well-suited for the synthesis of $\alpha, \alpha^{\prime}$-trans-disubstituted compounds. ${ }^{37}$ The Evans asymmetric alkylation/RCM strategy, however, has become established as a successful protocol for the construction of medium-ring oxacyclic skeletons, both $\alpha, \alpha^{\prime}$-cis- and $\alpha, \alpha^{\prime}$-trans-disubstituted, as illustrated with oxocenes in Scheme 29.

We had been successful at exploiting the versatility of the $\alpha$-alkoxy-substituted $N, N$-dimethylamide functionality and the substrate-controlled strategy, and thus we asked ourselves whether we could synthesize $\alpha, \alpha^{\prime}$-syn- or $\alpha, \alpha^{\prime}$ anti-disubstituted RCM substrates specifically without recourse to a chiral auxiliary. The embedded oxygen atoms highlighted in bold red in Scheme 29 suggested a way to employ what we call the 'protecting group dependent' al- 

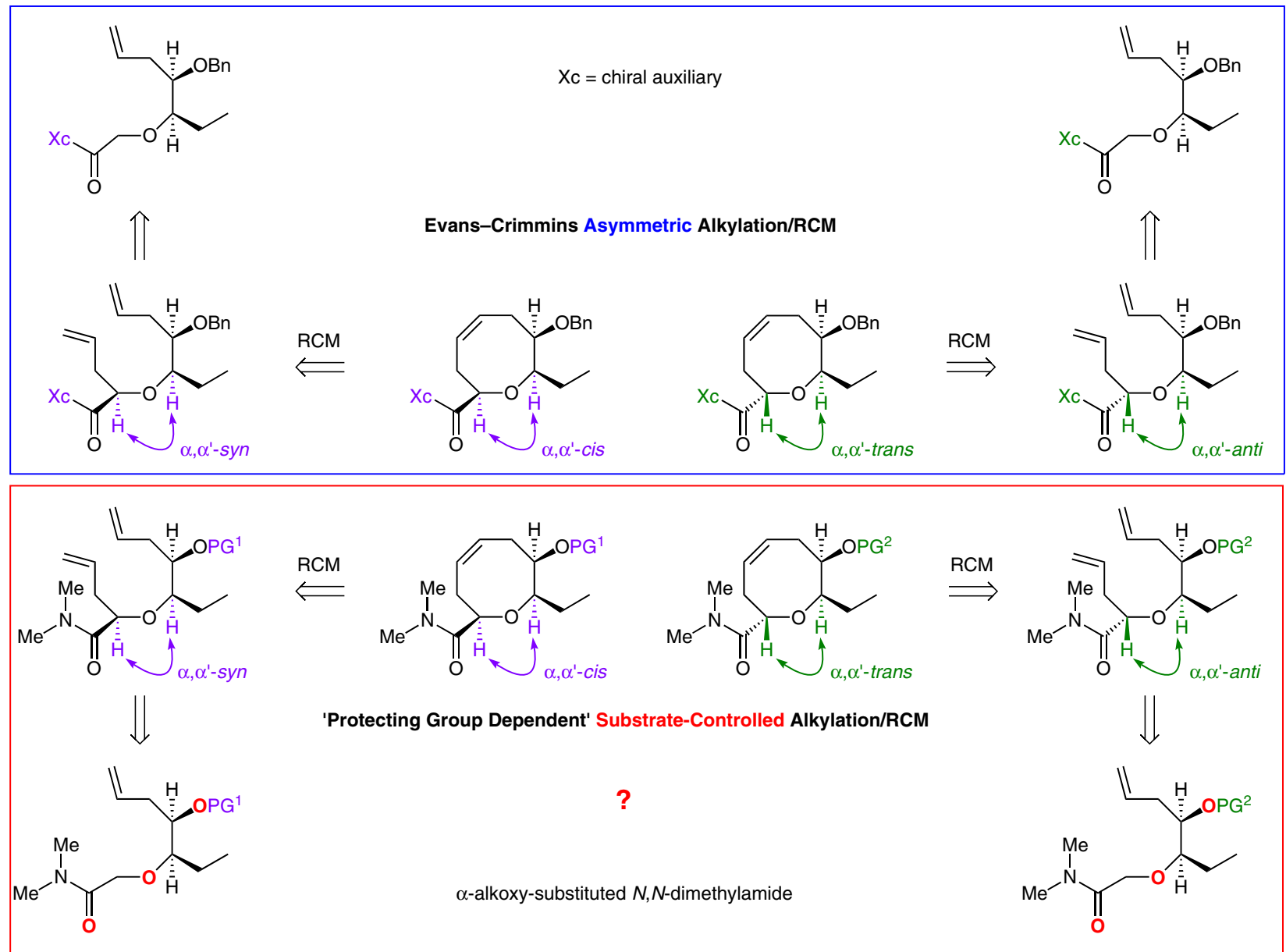

Scheme 29 Evans-Crimmins asymmetric alkylation/ring-closing metathesis versus protecting group dependent substrate-controlled alkylation/ring-closing metathesis

kylation/RCM strategy, which is essentially a substratecontrolled version of the Evans-Crimmins asymmetric alkylation/RCM strategy.

Itomanallene $\mathrm{A}, \mathrm{a}_{15}$ acetogenin with a 2,10-dioxabicyclo[7.3.0]dodecene skeleton, was isolated by the Suzuki group from Laurencia intricata in 2002 (Figure 3). ${ }^{38}$ The structure and absolute configuration of its two previously isolated diastereomeric congeners (-)-isolaurallene (77) $)^{39}$ and $(+)$-neolaurallene $(\mathbf{7 8})^{40}$ had already been firmly established based on X-ray crystallographic studies.

The relative stereochemistry of the bicyclic skeleton of itomanallene A was established through extensive spectroscopic studies. In particular, NOE interactions between H-6 and H-4 and between H-6 and H-7 were supportive of the assigned cis-relative stereochemistry of the tetrahydrofuran ring in the compound. Judging from the strong positive rotation of itomanallene A, its bromoallene moiety would be assigned as $S$ by the application of Lowe's rule. ${ }^{41}$ Because the relative stereochemistry between the dioxabicyclic skeleton and the bromoallene unit in itomanallene A could not be determined by spectroscopic methods, the two compounds 79a and 79b depicted in Figure 3 were proposed as possible structures of itomanallene A.

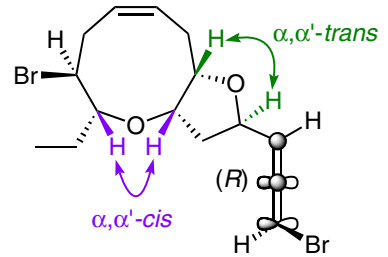

(-)-isolaurallene (77)

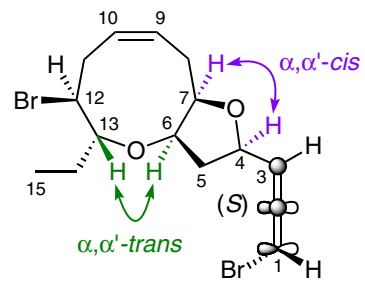

$$
79 a
$$$$
\text { two proposed structures of }(+) \text {-itomanallen }
$$

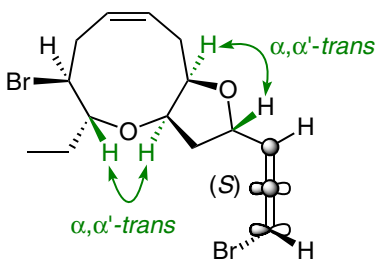

(+)-neolaurallene (78)

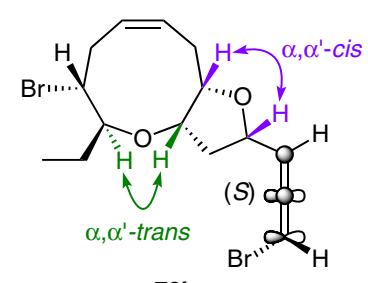

lene A

Figure 3 Representative medium-ring ether marine natural products with a 2,10-dioxabicyclo[7.3.0]dodecene skeleton

The structural features of this dioxabicyclic marine natural product that most appealed to us were the $\alpha, \alpha^{\prime}$-transdisubstituted oxonene and the $\alpha, \alpha^{\prime}$-cis-disubstituted tetra- 
hydrofuran. I would like to describe our asymmetric total synthesis and consequent structure revision of itomanallene A which epitomizes our completely substrate-controlled approach to the synthesis of $\alpha, \alpha^{\prime}$-transdisubstituted medium-ring oxacyclic natural products. ${ }^{42}$

Our first objective was to confirm the relative stereochemistry between the dioxabicyclic core and the bromoallene appendage of itomanallene A. For simplicity, we initially opted to synthesize ent-79a and 79b instead of the originally proposed structures 79a and 79b. This decision turned out to be a fortuitous one. Our retrosynthetic plan for the substrate-controlled synthesis of ent-79a and 79b, as shown in Scheme 30, once again hinged upon the versatility of the $\alpha$-alkoxy-substituted $N, N$-dimethylamide functionality.

We envisioned that bicyclic $\alpha$-alkoxy-substituted amide 81 could be elaborated to both ent-79a and 79b by installation of the respective bromoallene units through the intermediacy of bicyclic bromo-substituted aldehyde $\mathbf{8 0}$. We were confident that the $\alpha, \alpha^{\prime}$-cis-disubstituted tetrahydrofuran ring in bicyclic amide $\mathbf{8 1}$ could be constructed in a stereoselective manner through the IAEA reaction of tosylate 82, which in turn could be readily prepared from oxonene intermediate $\mathbf{8 3}$. We initially reasoned that the nine-membered ether ring $\mathbf{8 3}$ could be constructed by the RCM reaction of bis-alkene $\mathbf{8 4} .^{42}$

In this plan, the syn stereochemical relationship between $\mathrm{C}-12$ and $\mathrm{C}-13$ (corresponding to the final product) in RCM substrate 84 would be established by the manipulation of the $\alpha$-alkoxy-substituted $N, N$-dimethylamide moiety in 85 using our direct ketone synthesis/L-Selectride reduction protocol. For the greater challenge represented by the pivotal $\alpha, \alpha^{\prime}$-anti stereochemistry of ether 85 , we envisaged that this would be addressed through the 'chemoselective chelation-controlled' intermolecular amide enolate alkylation of $\mathbf{8 6}$. Further analysis suggested that alkylation substrate $\mathbf{8 6}$ would be readily accessible from known 4-methoxybenzyl (PMB) protected $(S)$-glycidol 88 via the installation of the C-6/C-7-syn stereochemistry by chelation-controlled nucleophilic addition to aldehyde $\mathbf{8 7}$.

A significant feature of this approach is that it showcases the versatility of $\alpha$-alkoxy-substituted amide enolate alkylation in establishing both sets of relative $\alpha, \alpha^{\prime}$-oxymethine configurations without recourse to additional chiral auxiliaries.

Scheme 31 presents a summary of our synthesis and structural revision of $(+)$-itomanallene A and our synthesis of ent-neolaurallene, with particular emphasis on the most salient stereo-, regio-, and chemoselective steps.

To this end, the C-6/C-7-syn stereochemistry in key alkylation substrate $\mathbf{8 6}$ was established in the presence of an $\alpha-$ alkoxy-substituted amide through the chemoselective chelation-controlled nucleophilic addition of allyltributylstannane to aldehyde 87, derived from known PMB-protected $(S)$-glycidol 88, ${ }^{43 a}$ using the method of Keck. ${ }^{44}$
The significant challenge of achieving the critical $\alpha, \alpha^{\prime}-$ anti stereochemistry in $\alpha$-alkoxy-substituted amide $\mathbf{8 5}$ was successfully addressed by the chemoselective chelation-controlled intermolecular amide enolate alkylation of 86. In this scenario, the alkylating agent approaches the less-hindered convex face of cup-shaped chelated enolate intermediate $\mathbf{E}$, in which the bulky group at C-6 prefers to be located, to afford the desired product $\mathbf{8 5}$ as essentially a single isomer. ${ }^{45}$ It is worth noting that the PMB-protected hydroxy group participates in the chelation in preference to the triisopropylsilyl (TIPS) protected one, which is known to be a poor coordinating group.

As planned, the syn stereochemical relationship between C-12 and C-13 in RCM substrate 84 was established by the manipulation of the $\alpha$-alkoxy-substituted $N, N$-dimethylamide in 85 through the intermediacy of ketone $\mathbf{8 9}$ using our direct ketone synthesis/L-Selectride reduction protocol. ${ }^{21,27}$
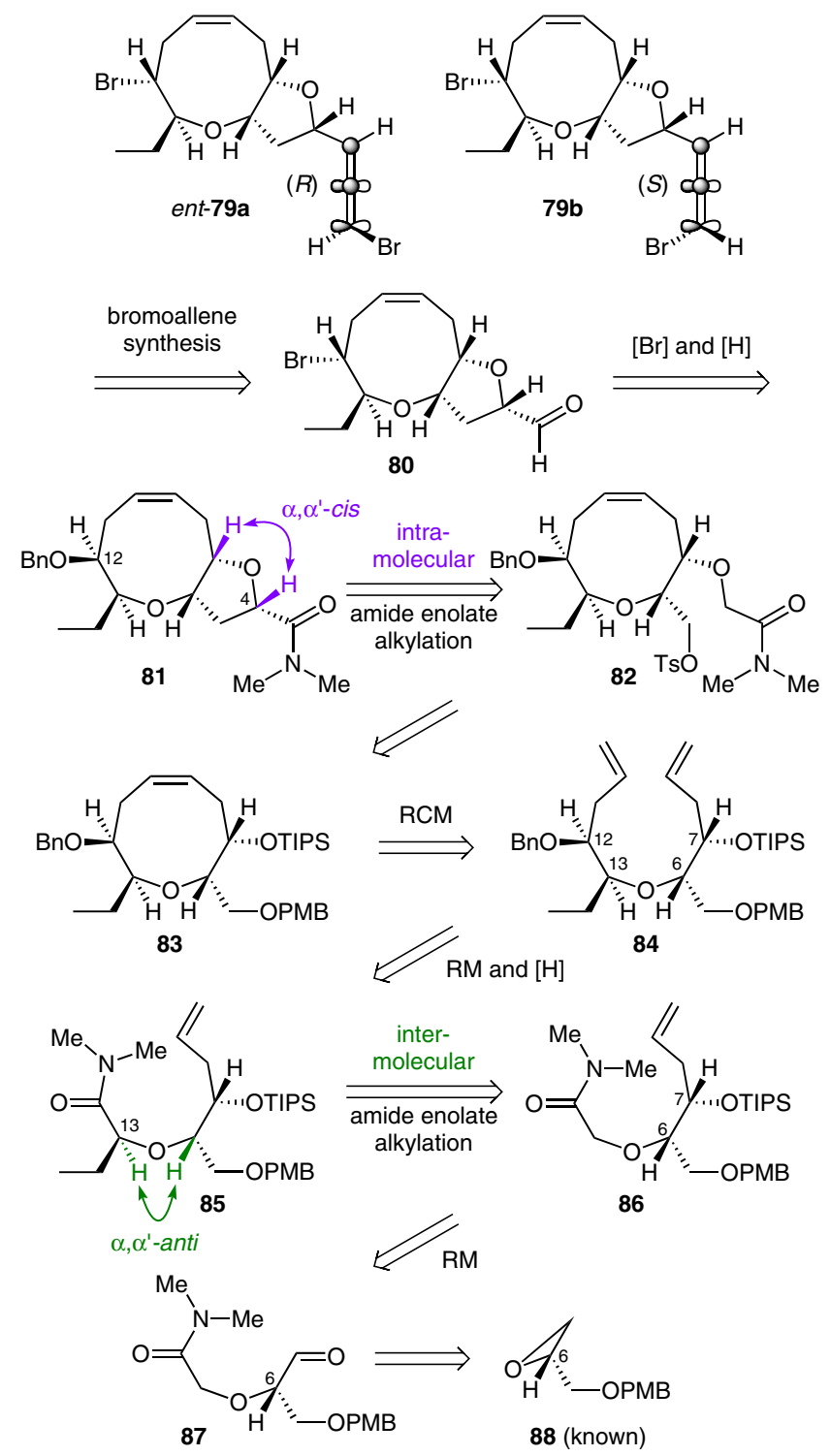

Scheme 30 Retrosynthetic plan for compounds ent-79a and 79b 


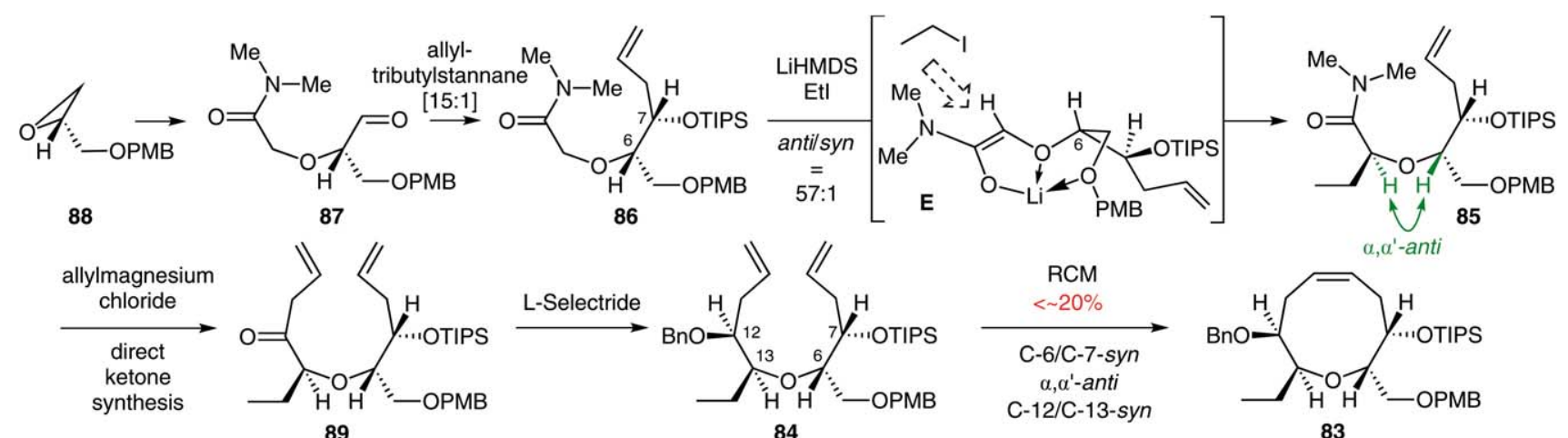

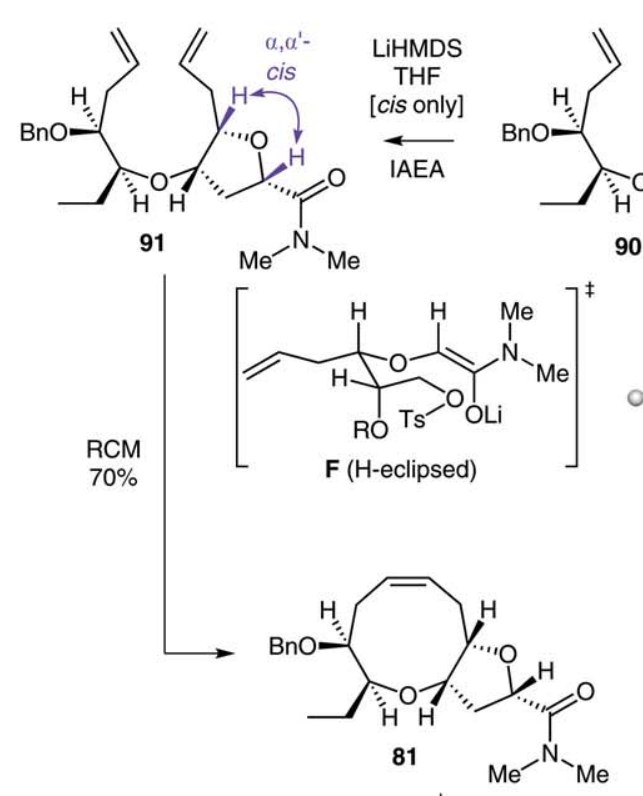

$[\mathrm{Br}]$ and 'ate' complex

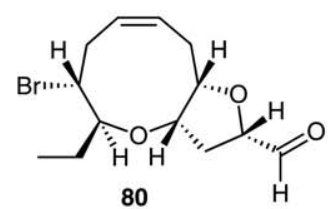

$(i-\mathrm{PrO})_{3} \mathrm{Ti}=\mathrm{TMS}$

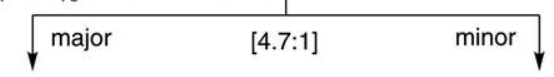

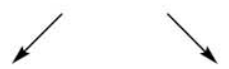

ل LiHMDS, toluene

83

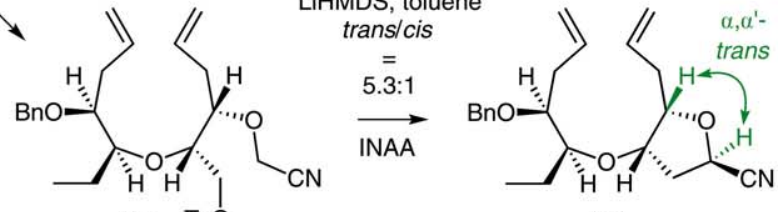
90' TsO

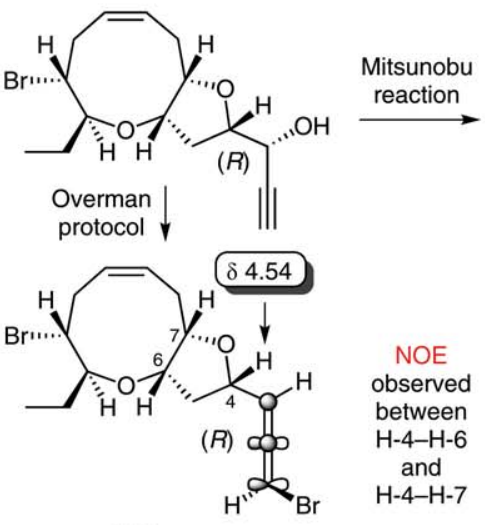

ent-79a

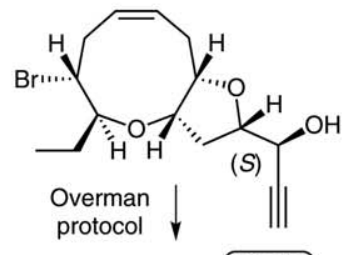

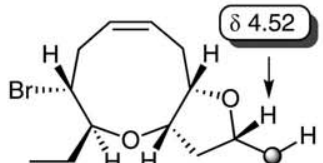

(S) 000 Br" $^{\prime \prime}$

$79 b$

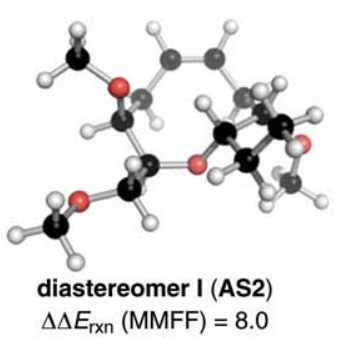

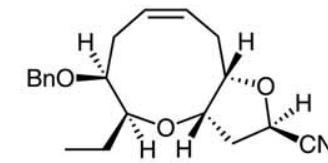

81'

$[\mathrm{Br}]$ and DIBAL-H

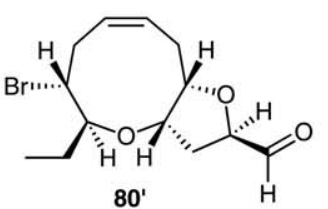

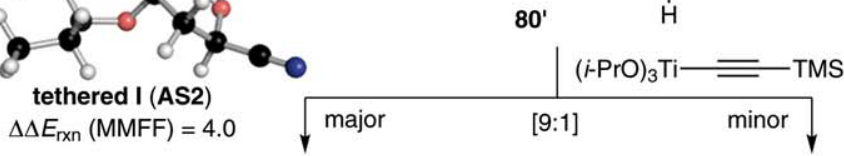

$\delta 4.86$
$H-4$

natural itomanallene $\mathrm{A}$

\&

$\mathrm{H}-4$

natural neolaurallene

Scheme 31 Summary of the asymmetric total synthesis and structure revision of (+)-itomanallene A 
To our great disappointment, upon conducting the hitherto uninvestigated RCM of C-6/C-7-syn, $\alpha, \alpha^{\prime}$-anti,C-12/C-13syn bis-alkene 84, only a low yield of the desired oxonene $83(\sim 20 \%)$ was obtained despite a considerable amount of effort. The rationale that a cyclic constraint might facilitate the RCM prompted us to construct the five-membered ring first. To this end, the $\alpha, \alpha^{\prime}$-cis-disubstituted tetrahydrofuran ring was constructed in a stereoselective manner through the IAEA reaction of tosylate $\mathbf{9 0}$ as anticipated, probably via $\mathrm{H}$-eclipsed transition state F. Gratifyingly, the resulting tetrahydrofurano-fused diene 91 underwent RCM using Grubbs' second generation catalyst to produce key bicyclic oxonene $\mathbf{8 1}$ in $70 \%$ yield.

When facing obstacles throughout my career, such as the above-mentioned inefficient RCM, I have frequently overcome them using hunch-based reasoning rather than well thought out chemical rationale. On the plus side, this alchemical journey has often led to serendipitous discoveries. When I could not properly rationalize these, circumstances led me to use vague expressions, such as 'might', 'probably', 'presumably', and 'we are still developing a rationale for' in my publications. As a result, very keen reviewers occasionally gave me horrendous scores on 'scholarly presentation'. Recently, I have had the wonderful and extremely fortunate opportunity to collaborate with a superb young computational chemist, Dr. Robert S. Paton (University of Oxford). Nowadays, I can walk the streets of Seoul with my head held high, exuding a confidence which used to be foreign to my chemical life.

It was known that the relative stereochemistry of the incipient cyclic ether oxygen atom with respect to each of the adjacent oxygen substituents, as well as the $\alpha, \alpha$ '-relative stereochemistry, could exert subtle conformational effects on the rate of the RCM to give oxonenes. ${ }^{42,46}$ To gain insight into these conformational effects, Dr. Paton embarked on computational studies of the RCM reactions of the eight possible diastereomers of 84, and for (Z)2,3,4,7,8,9-hexahydrooxonine he located the global minimum AS2 conformation $\mathbf{G}$, as depicted in Scheme $31 .{ }^{47}$

While simplifying the process by turning protecting groups into methyl groups in the model, the stabilities of the open chain (i.e., with terminal alkenes) and ringclosed forms (following RCM) of the eight possible diastereomers of $\mathbf{8 4}$ were calculated in an attempt to gauge the effect of stereochemistry on the energetics of the RCM. The mechanism of ring closure involves ruthenium-alkylidene and metallocycle intermediates and transition states, but the quantum chemical calculations required to study these species being such flexible systems are computationally intractable.

Thus, we focused instead on the relative stabilities of the diastereomeric reactants and cyclized products to give values for $\Delta \Delta E_{\mathrm{rxn}}$; this enabled us to rank the systems in terms of thermodynamic favorability. The open-chain reactant energies all lie within $0.5 \mathrm{kcal} / \mathrm{mol}$ of each other because the reactants are flexible and may adopt a number of conformations. Following RCM, however, the cyclized product stability is more variable, and it is this energy that dictates the $\Delta \Delta E_{\mathrm{rxn}}$ value.

With the exception of one diastereomer, all the systems showed a preference for the asymmetric AS2 conformation. In AS2 conformation $\mathbf{G}$, preferentially adopted by the ring, one of the dihedral angles $\left(\Theta_{\mathrm{CCCH}}=20.8^{\circ}\right)$ is partially eclipsing (Scheme 31 ). In nearly all of the systems, a $\beta$-alkoxy group pointed away from the ring, presumably to avoid destabilization from an eclipsing CCCO interaction. In $\alpha, \alpha^{\prime}$-trans-disubstituted diastereomer $\mathbf{I}$, which corresponds to the stereochemistry of $\mathbf{8 4}$, the eclipsing $\mathrm{CCCO}$ interaction is present and results in the destabilization of this structure and retardation of the ring closure. Diastereomer I had less favorable reaction energy in comparison with the other diastereomers - how unlucky we were that our RCM substrate happened to be the worst system! - and this analysis is consistent with the fact that the attempted RCM of $\mathbf{8 4}$ proceeded in $<20 \%$ yield.

However, we also modeled furano-fused diene 91' with the same stereochemistry as $\mathbf{8 4}$, but in which the alkoxy side chains are tethered together. This form, tethered I, was computer modeled to cyclize with a more favorable reaction energy (by 3-4 kcal/mol) compared with that of diastereomer I. This was in agreement with the experimental, where the RCM reactions of 91 and 91' proceeded in 70 and $57 \%$ yield, respectively, after the side chains of their precursors had been tethered.

Bicyclic bromo-substituted aldehyde 80, derived from $\alpha$ alkoxy-substituted amide $\mathbf{8 1}$ using reduction with the diisobutylaluminum hydride (DIBAL-H) $/ n$-BuLi ate complex as a key step, ${ }^{48}$ could be elaborated to both ent-79a and 79b. This involved the installation of the respective bromoallene units via the well-established Overman protocol [titanium (trimethylsilyl)acetylide addition; trisylation; $\mathrm{Cu}$-catalyzed anti- $\mathrm{S}_{\mathrm{N}} 2^{\prime}$ reaction]. ${ }^{49}$ Unfortunately, the proton NMR spectra of both compounds were different from that of natural itomanallene A. In particular, the chemical shift values of the $\mathrm{C}-4$ protons were significantly different, as indicated in Scheme 31.

Incidentally, one of the questions I am asked most frequently after lecture presentations is about the use of the ate complex for the reduction of an $\alpha$-alkoxy-substituted amide functionality to give an aldehyde. Why not use DIBAL-H alone? The reason we have placed so much trust in this reagent is that it has never failed us. In addition, it was developed by a Korean chemist (Professor Sunggak Kim at KAIST, now at Nanyang Technical University) whom I greatly respect, so this could also be an example of a branch of chemopolitics called 'patriotic chemistry'.

Careful examination of NMR spectroscopic data from structurally related natural products containing a bromoallene led to the conclusion that itomanallene A might possess an $\alpha, \alpha^{\prime}$-trans-disubstituted tetrahydrofuran, despite the aforementioned reported observation of an NOE interaction between H-4 and H-6. Based on this assumption, we set out to synthesize the C-4 epimers of ent-79a 
and $79 b$. Incidentally, the C-4 epimer of ent-79a corresponds to the enantiomeric form of neolaurallene (ent-78).

To this end, the requisite $\alpha, \alpha^{\prime}$-trans-disubstituted tetrahydrofuran stereochemistry could be secured by the formation of major isomer 91' upon exposure of cyano tosylate $\mathbf{9 0}^{\prime}$ to lithium hexamethyldisilazide (LiHMDS) using the INAA methodology based on the pioneering work of Stork and co-workers ${ }^{50}$ and subsequent investigations by Fleming and co-workers. ${ }^{51}$

Many of the extant applications of this INAA methodology have involved the stereoselective construction of a quaternary center by taking advantage of the relatively small size and powerful nucleophilicity of the nitrile functionality. However, the presence of an acidic proton in $\alpha-$ unsubstituted cases, such as the above, raises a concern regarding the potential loss of the stereochemical integrity during the cyclization.

An equilibration experiment to probe the relative stability of the isomers showed that the $\alpha, \alpha^{\prime}$-trans-isomer is more stable than the corresponding cis one by a 2:1 margin. Furthermore, from a deuterium incorporation study as well as the separate resubjection of the isolated isomers to the reaction conditions, we were able to establish that the selectivity ratio of INAA arises from a kinetically controlled process under the cyclization conditions. Internal alkylation of the linear, N-metalated nitrile anion generated under the reaction conditions gave the desired $\alpha, \alpha^{\prime}$ trans-disubstituted tetrahydrofuran as the major product. We reasoned that the intermediate passes through the geometry of transition state $\mathbf{H}$, which benefits from stereoelectronic stabilization by placing the carbon-nitrile bond antiperiplanar to an oxygen lone pair on the ether oxygen. $^{47,52}$

Intermediate $91^{\prime}$ was then transformed into $79 \mathrm{c}$ and ent-78 in an analogous manner to derivative 91, except that in this case aldehyde intermediate $\mathbf{8 0}^{\prime}$ was derived by reduction of the nitrile function with DIBAL-H.

The spectroscopic characteristics and optical rotation of our synthetic material 79c, corresponding to a C-4 epimer of one of the proposed structures, compound $\mathbf{7 9 b}$, were in good agreement with those reported for natural $(+)$-itomanallene A. Based on this synthesis, the structure of $(+)$-itomanallene A should be revised to that shown in Scheme 31. In addition, the spectroscopic and optical rotation data for our synthetic compound C-4-epi-ent-79a, corresponding to ent-78, were in close agreement with those of natural neolaurallene with the exception of the sign for the optical rotation.

\section{6}

\section{General Synthetic Plan for Dioxabicyclic Bromoallene Marine Natural Products Having either a 2,10-Dioxabicyclo[7.3.0]do- decene or 2,9-Dioxabicyclo[6.3.0] undecene Skeleton}

The above-described first asymmetric total synthesis and consequent structure revision of $(+)$-itomanallene A provides a versatile strategy for the synthesis of both $\alpha, \alpha^{\prime}-c i s-$ and $\alpha, \alpha^{\prime}$-trans-disubstituted tetrahydrofurans in such dioxabicylic marine natural products and related structures through the judicious choice of an amide enolate versus a nitrile anion, respectively, for the intramolecular alkylation. It is worth mentioning that it was our casual interest in the comparative stereoselectivity of branched ester enolates versus linear nitrile anions in intramolecular alkylations that launched a campaign that first began with our unintended synthesis of brefeldin A (see Section 2), and more than a decade later blossomed into a general strategy for the construction of both $\alpha, \alpha^{\prime}$-cis- and $\alpha, \alpha^{\prime}$-trans-disubstituted tetrahydrofurans in dioxabicylic marine natural products.

Based on the insights acquired during our synthesis and structure revision of itomanallene A (79c), and employing as a common starting material glycidol, which is commercially available in both enantiomeric forms, we designed a general synthetic strategy to encompass the creation of any arbitrary relative $\alpha, \alpha^{\prime}$-oxymethine configurations in any of 32 biogenetically plausible stereoisomeric dioxabicyclic bromoallene marine natural products having either the 2,10-dioxabicyclo[7.3.0]dodecene or 2,9-dioxabicyclo[6.3.0] undecene skeleton. Scheme 32 shows our general synthetic plan for the half of the 32 isomeric natural products that are derived from $(S)$-glycidol. This might be nearly illegible given the minute fonts used; the other half derived from enantiomer $(R)$-glycidol are not shown because of space limitations.

I am really good at and fond of drawing these schemes in particular complicated ones, such as Scheme 32. On one occasion, I showed off the two schemes covering all 32 isomers to my student, but she seemed unappreciative. It was then that I realized she thought I intended that she synthesize all of them, and her scowl meant that she viewed my masterpieces as a life sentence of hard labor!

Since we had developed optional access to $\alpha, \alpha^{\prime}$-cis- and $\alpha, \alpha^{\prime}$-trans-disubstituted tetrahydrofurans based on using IAEA and INAA protocols, respectively, all we needed for the execution of the general scheme was to secure the medium-sized ring $\alpha, \alpha^{\prime}$ stereochemistry complementary to that obtained via the above-mentioned chemoselective chelation-controlled alkylation $(\mathbf{8 6} \rightarrow \mathbf{8 5}$, see Section 5$)$. To this end, we pinned our initially confident hopes for a solution on our 'dianion' alkylation methodology, as depicted in Scheme 33. 
General Synthetic Strategy

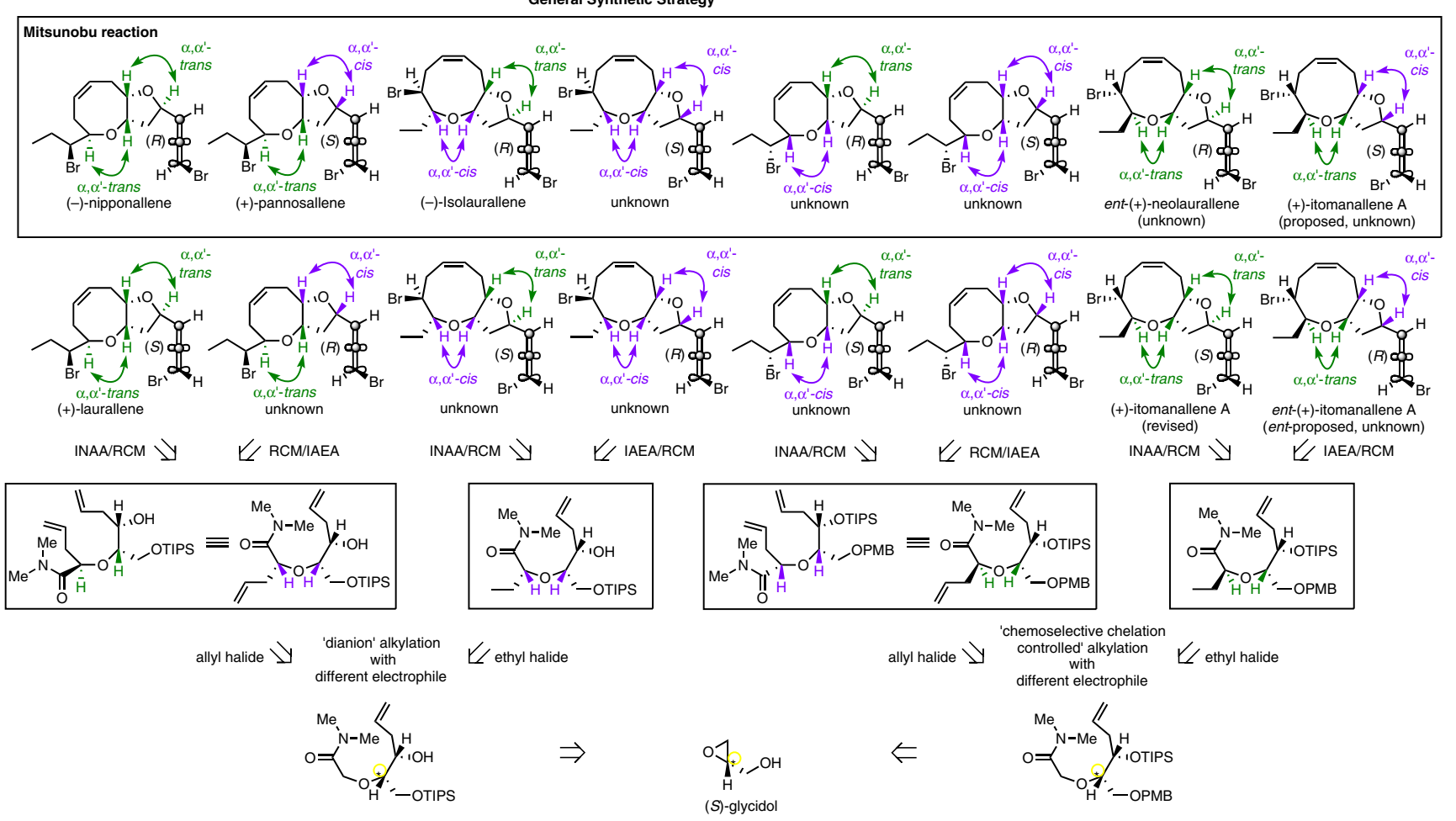

Scheme 32 General synthetic plan for 16 biogenetically plausible dioxabicyclic bromoallene marine natural products having either a 2,10dioxabicyclo[7.3.0]dodecene or 2,9-dioxabicyclo[6.3.0] undecene skeleton from $(S)$-glycidol

The dianion alkylation of hydroxy-containing $\alpha$-alkoxysubstituted amides 92a-d having various substituents at $\mathrm{C}-6$ (based on the final natural product) $[\mathrm{R}=\mathrm{Et}$, $\mathrm{CH}_{2} \mathrm{CH}_{2} \mathrm{OBn}, \quad \mathrm{CH}_{2} \mathrm{CH}_{2} \mathrm{CH}_{2} \mathrm{OBn}, \quad$ and $\mathrm{CH}_{2} \mathrm{CH}\left(\mathrm{OCH}_{2} \mathrm{CH}_{2} \mathrm{O}\right)$, respectively] offered serviceable syn/anti stereoselectivity (ca. 6-9:1) (Scheme 33). We have utilized this reaction as a key step for the substratecontrolled total synthesis of medium-ring oxacyclic natural products in our laboratories. ${ }^{28,53}$ The observed stereoselectivity could be rationalized by empirical model $\mathbf{I}$, with the electrophile approaching the least-hindered side of the $\mathrm{H}, \mathrm{H}$-eclipsed conformation.

To our surprise and disappointment, our initial effort to establish the desired complementary $\alpha, \alpha^{\prime}$-cis-disubstituted oxonene stereochemistry via dianion alkylation of PMB-protected $\alpha$-alkoxy-substituted amide 92e $(\mathrm{R}=$ $\left.\mathrm{CH}_{2} \mathrm{OPMB}\right)$ gave very disappointing stereoselectivity (syn/anti $=1.1: 1$ ) and chemical yield $(54 \%) .{ }^{47} \mathrm{It}$ is worth mentioning that the triisopropylsilylation of 92e provided substrate 86, required for the chemoselective chelationcontrolled intermolecular amide enolate alkylation in our earlier described synthesis of (+)-itomanallene A (79c).$^{42}$ The use of 92e in this sequence would have simplified our general approach to our target compounds, but the virtual absence of stereoselectivity in the key step reminded us of the old adage, "The best laid schemes of mice and men go often awry, and leave us nothing but grief and pain for promised joy!"

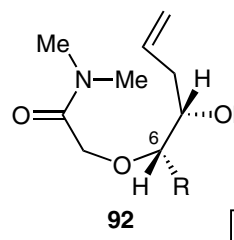

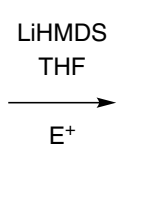

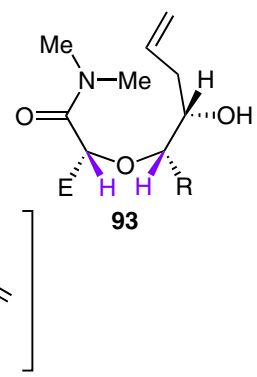

\begin{tabular}{|c|c|c|c|}
\hline $\mathrm{R}$ & Substrate no. & $\mathrm{E}^{+}$ & $\begin{array}{c}\text { Ratio } \\
\text { (synlant) }\end{array}$ \\
\hline $\mathrm{Et}$ & $\mathbf{9 2 a}$ & allyl bromide & $9.3: 1$ \\
\hline $\mathrm{CH}_{2} \mathrm{CH}_{2} \mathrm{OBn}$ & $\mathbf{9 2 b}$ & allyl bromide & $6: 1$ \\
\hline $\mathrm{CH}_{2} \mathrm{CH}_{2} \mathrm{CH}_{2} \mathrm{OBn}$ & $\mathbf{9 2 c}$ & allyl bromide & $6: 1$ \\
\hline $\left.\mathrm{CH}_{2} \mathrm{CH}_{(\mathrm{OCH}} \mathrm{OCH}_{2} \mathrm{O}\right)$ & $\mathbf{9 2 d}$ & allyl iodide & $6.3: 1$ \\
\hline $\mathrm{CH}_{2} \mathrm{OPMB}$ & $\mathbf{9 2 e}$ & ethyl iodide & $1.1: 1$ \\
\hline $\mathrm{CH}_{2} \mathrm{OTIPS}$ & $\mathbf{9 2 f}$ & ethyl iodide & $37: 1$ \\
\hline
\end{tabular}

Scheme 33 Stereoselectivity in dianion alkylation

We reasoned that the use of the corresponding TIPS-protected hydroxymethyl group might avoid any potential interference owing to chelation because TIPS ethers are known to be poor coordinating groups, unlike their PMB derivatives. We were delighted to find that the application of our protocol to TIPS-protected $\alpha$-alkoxy-substituted amide 92f $\left(\mathrm{R}=\mathrm{CH}_{2} \mathrm{OTIPS}\right)^{47}$ proved successful, and 
treatment with LiHMDS in the presence of ethyl iodide gave the desired $\alpha, \alpha^{\prime}$-syn-disubstituted ether 93f with excellent stereoselectivity (syn/anti $=37: 1 ; 91 \%$ total yield). Although I dread to write it this way, for reasons I have alluded to earlier, we are still developing a rationale to explain this relatively high degree of stereoselectivity.

Incorporating the established methodologies for creating any arbitrary relative $\alpha, \alpha^{\prime}$-oxymethine configurations, the more sensible and easily legible Scheme 34 illustrates our entirely substrate-controlled sequences from $(S)$-glycidol (97) to some of those marine natural products among the 32 having either the 2,10-dioxabicyclo[7.3.0]dodecene or 2,9-dioxabicyclo[6.3.0] undecene skeleton which have been isolated from natural sources to date. ${ }^{47}$ The compounds with the former skeleton are (-)-isolaurallene (77),${ }^{39}$ the enantiomeric form of natural $(+)$-neolaurallene (ent-78), ${ }^{40}$ and (+)-itomanallene A (79c) ${ }^{38}$ while those with the latter skeleton are (+)-laurallene $(\mathbf{9 4}),{ }^{54}(-)$-nipponallene (the bromoallene diastereomer of laurallene; not shown in Scheme 34), ${ }^{55}$ and (+)-pannosallene (95). ${ }^{56}$

Our general approach to establish the $\alpha, \alpha^{\prime}$-relative stereochemistry of the medium-sized ring (oxonene or oxocene) and tetrahydrofuran involved the judicious pairing of our protecting group dependent intermolecular amide enolate alkylation (either chemoselective chelation-controlled or dianion alkylation) with either our intramolecular amide enolate or intramolecular nitrile anion alkylation. The application of this approach offered us optional access to any of these dioxabicyclic bromoallene marine natural products. The requisite bromoallene appendage could be elaborated via application of the stereoselective Overman protocol to either the corresponding propargylic alcohol or its diastereomer, obtained using a Mitsunobu inversion reaction.

More specifically, our chemoselective chelation-controlled intermolecular amide enolate alkylation $(\mathbf{8 6} \rightarrow \mathbf{8 5})$ and INAA were utilized to establish the $\alpha, \alpha^{\prime}$-trans-disubstituted oxonene and tetrahydrofuran stereochemistry, respectively, in itomanallene A (79c) and ent-(+)neolaurallene (ent-78). Conversely, the intermolecular dianion alkylation strategy $(\mathbf{9 2 f} \rightarrow \mathbf{9 3 f})$ and INAA provided access to the $\alpha, \alpha^{\prime}$-cis-disubstituted oxonene skeleton and $\alpha, \alpha^{\prime}$-trans-disubstituted tetrahydrofuran, respectively, for the synthesis of (-)-isolaurallene (77). In addition, the use of an allyl halide electrophile in place of an ethyl halide for the intermolecular dianion alkylation $(\mathbf{9 2 f} \rightarrow \mathbf{9 6})$ enabled us to construct the $\alpha, \alpha^{\prime}$-trans-disubstituted oxocene skeleton in (+)-laurallene (94) and (+)-pannosallene (95). The $\alpha, \alpha^{\prime}$-trans- and $\alpha, \alpha^{\prime}$-cis-disubstituted tetrahydrofuran stereochemistry in these two products was established through intramolecular nitrile anion and intramolecular amide enolate alkylation, respectively (Scheme 34).

An asymmetric total synthesis of (-)-isolaurallene (77) was accomplished in 22 steps from readily available TIPS-protected $(S)$-glycidol, ${ }^{43 \mathrm{~b}}$ as summarized in Scheme 35 with particular emphasis on the stereoselectivity. ${ }^{47}$ Tracing the path from the smallest stars to the largest, we employed chelation-controlled nucleophilic addition, in-

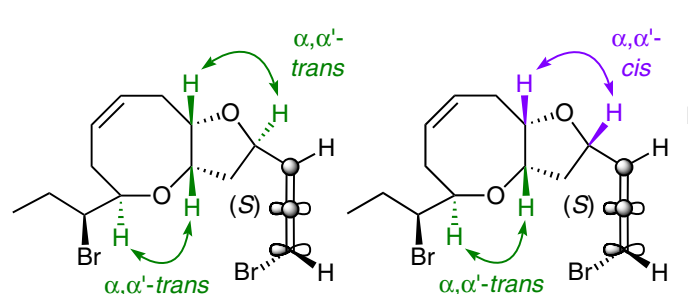

(+)-laurallene (94) INAA/RCM \y (+)-pannosallene (95) RCM/IAEA/ 1/ $\begin{gathered}\text { Mitsunobu } \\ \text { reaction }\end{gathered}$<smiles>C=CC[C@H](O[C@@H](C[18OH])CO[OH+])C(=O)N(C)C</smiles>

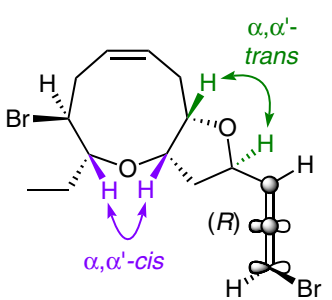

(-)-isolaurallene (77) INAA/RCM/ Mitsunobu reaction

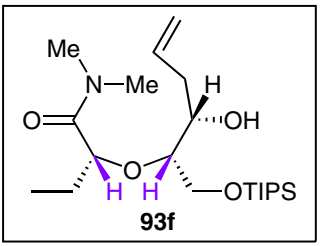

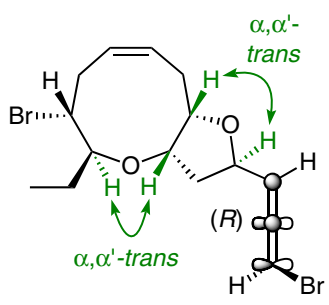

ent-(+)-neolaurallene (ent-78) INAA/RCM/ Mitsunobu \y reaction

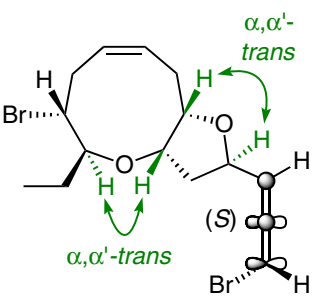

(+)-itomanallene A (79c)

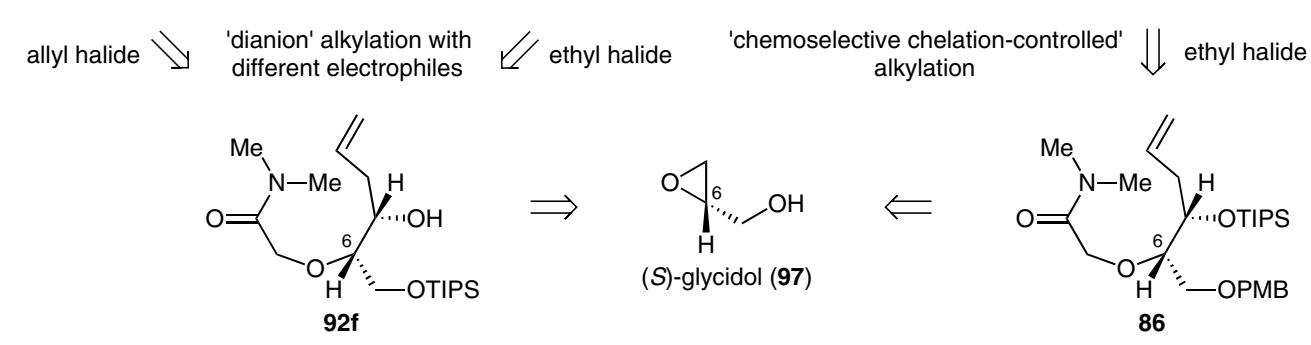

Scheme 34 General synthetic plan for known dioxabicyclic bromoallene marine natural products having either the 2,10-dioxabicyclo[7.3.0]dodecene or 2,9-dioxabicyclo[6.3.0]undecene skeleton from $(S)$-glycidol (97) 
termolecular amide enolate alkylation, L-Selectride reduction, INAA, and the Overman protocol. ${ }^{57}$ Most importantly, in our completely substrate-controlled synthesis, an intermolecular dianion alkylation and INAA sequence provided access to the $\alpha, \alpha^{\prime}$-cis-disubstituted oxonene skeleton and $\alpha, \alpha^{\prime}$-trans-disubstituted tetrahydrofuran, respectively.

Of note here is that the RCM of bis-alkene 98 proceeded with high efficiency as anticipated (96\% yield). Synthetic intermediates with stereochemistry corresponding to that of 98 have been observed experimentally to undergo RCM in good yields (Crimmins' isolaurallene synthesis), and this has been attributed to a reinforcing double gauche effect. $^{46 a, b}$ The reaction energy of diastereomer II, which stereochemically corresponds to $\mathbf{9 8}$, was computed to be the second most favorable among those of the previously mentioned eight possible diastereomers. This is predominantly due to the minimization of torsional strain, as discussed previously in connection with our itomanallene A synthesis (see Scheme 31), while benefitting from a single gauche effect. In fact, although diastereomer III (epimeric at C-7 to diastereomer II) does not benefit from any gauche carbon-oxygen bond interactions, it was computed to be the most stable ring-closed product. Furthermore, a comparative RCM experiment supported our computa-
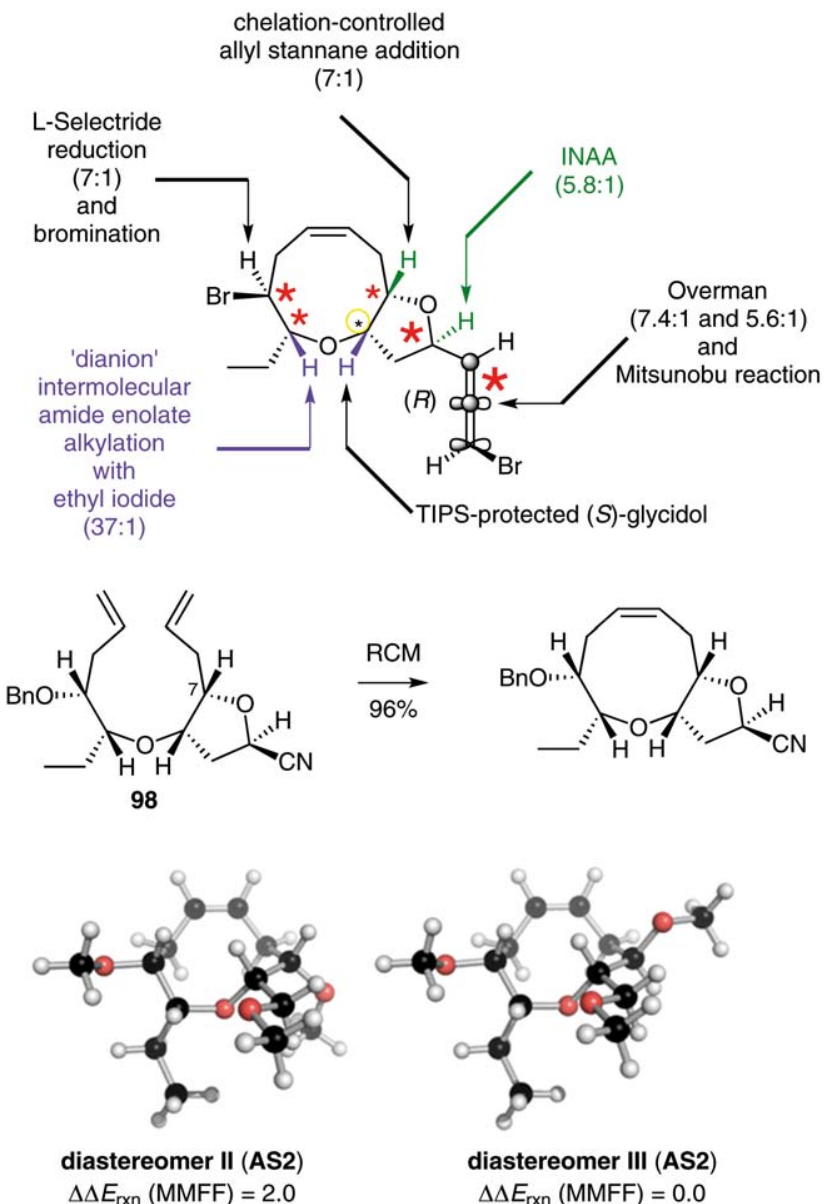

$\Delta \Delta E_{\mathrm{rxn}}(\mathrm{MMFF})=2.0$

$\Delta \Delta E_{\mathrm{rxn}}(\mathrm{MMFF})=0.0$

Scheme 35 Highlights of the asymmetric total synthesis of (-)-isolaurallene (77)

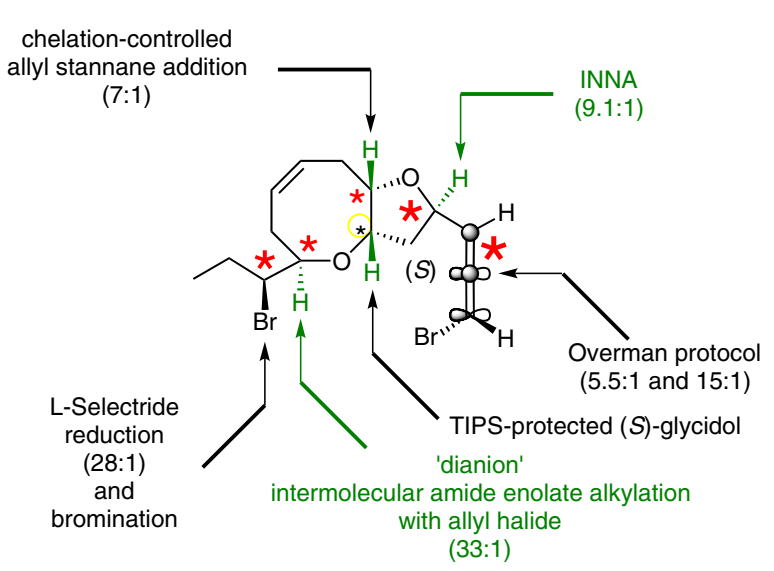

Scheme 36 Highlights of the asymmetric total synthesis of (+)-laurallene (94)

tional prediction of the greater stability of diastereomer III and our new rationalization of the reactivity based on the minimization of torsional strain in the preferred AS2 ring conformation.

Likewise, a highly stereoselective and efficient 21-step synthesis of (+)-laurallene (94) was accomplished in an entirely substrate-controlled fashion starting from the known TIPS-protected $(S)$-glycidol (Scheme 36). ${ }^{43 \mathrm{~b}}$ Our strategy is highly adaptable in that either an $\alpha, \alpha^{\prime}$-cis-disubstituted oxonene or $\alpha, \alpha^{\prime}$-trans-disubstituted oxocene product can be accessed by employing either an ethyl or allyl halide, respectively, as the electrophile for the dianion alkylation. ${ }^{47}$

Finally, the first asymmetric total synthesis of (+)-pannosallene (95) was accomplished in a substrate-controlled fashion in 19 steps from TIPS-protected $(S)$-glycidol, ${ }^{43 \mathrm{~b}}$ as illustrated in Scheme 37. In addition, our synthesis established the relative stereochemistry between the dioxabicyclic skeleton and the bromoallene unit in $\mathbf{9 5}$. Our concise synthesis of this $\mathrm{C}_{15}$ acetogenin containing the rare $\alpha, \alpha^{\prime}$ cis-disubstituted tetrahydrofuran moiety features highly stereoselective intermolecular and intramolecular amide enolate alkylations as key steps to establish both sets of relative $\alpha, \alpha^{\prime}$-oxymethine configurations in the product. ${ }^{47}$

Scheme 38 summarizes our work on the construction of medium-ring oxacyclic skeletons and highlights and compares the RCM and IAEA approaches using the cases of the $\alpha, \alpha^{\prime}$-cis-and $\alpha, \alpha^{\prime}$-trans-disubstituted oxocenes. It is known that these oxocenes can be constructed by RCM, which is one of the most efficient methods available for the purpose. We believe that our major contribution to this field has been demonstrating the feasibility of constructing $\alpha, \alpha^{\prime}$-cis-disubstituted medium-sized rings via IAEA.

Our IAEA strategy, which admittedly has a more limited scope compared with RCM, still possesses a distinct advantage over it in certain cases. For instance, we can construct $(Z)$ - as well as (E)-oxonenes specifically, as previously illustrated in our synthesis of $(-)$-cladiella6,11-dien-3-ol (65). ${ }^{33}$ Furthermore, the oxocene core in bermudenynol (99), which also contains a vinyl chloride moiety, could be constructed using our IAEA methodolo- 


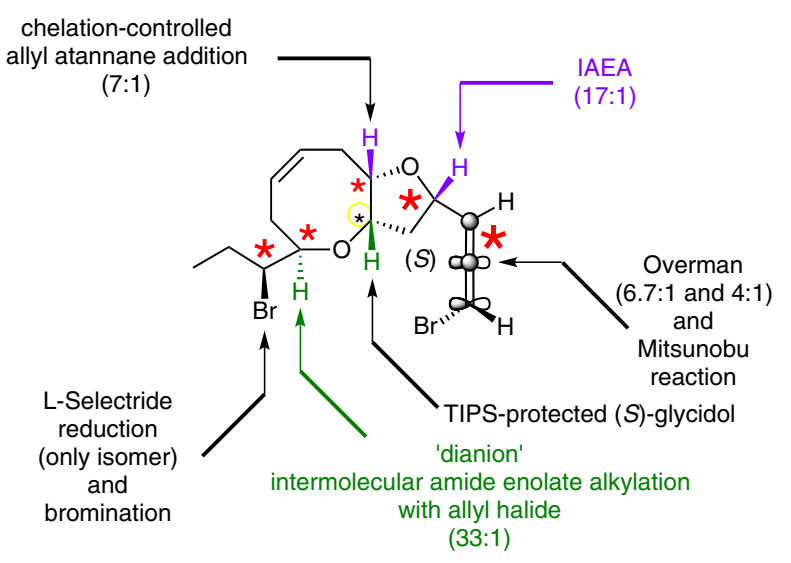

Scheme 37 Highlights of the asymmetric total synthesis and structure confirmation of $(+)$-pannosallene (95)

gy ${ }^{58}$ in comparison, the construction of vinyl chloride containing oxocenes via RCM is problematic, as has been alluded to by Steve Weinreb. ${ }^{59}$ In addition, we have shown that the requisite RCM substrates can be synthesized in a substrate-controlled fashion via intermolecular amide enolate alkylation that avoids the use of additional chiral auxiliaries through the judicious choice of protecting groups.

\section{$7 \quad$ Conclusion}

In conclusion, taking inspiration from the steroidal transhydrindane problem, we have developed a general strategy for the stereoselective construction of cycloalkanecarboxylates involving a folding and allylic strain controlled intramolecular ester enolate alkylation, and we have applied this to total syntheses of natural products of modest complexity. En route to the corresponding $\mathrm{S}_{\mathrm{N}} 2^{\prime}$ version, we made a serendipitous discovery that ultimately led to what we call the olefin geometry dependent IAEA. Application and extension of this methodology allowed us to achieve completely substrate-controlled, asymmetric total syntheses of diverse medium-ring oxacyclic marine natural products. Along the way, fortuitous discoveries using organoselenium-based methods led to intriguing biomimetic syntheses of Laurencia metabolites.

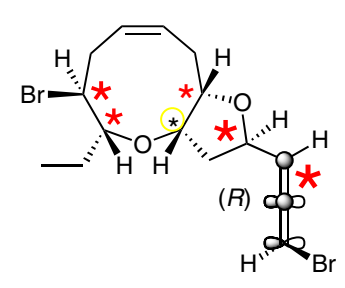

Kim $\Downarrow 22$ steps<smiles>[H][Y6]1([O-])CO1</smiles>

$\star$ : source of absolute chirality

* : by substrate control

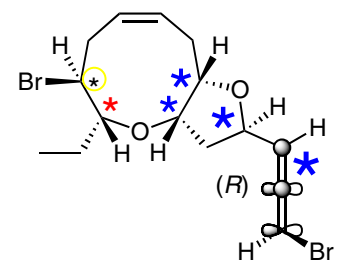

28 steps $\Downarrow$ Crimmins<smiles>O=C(COBr)N1C(=O)OC[C@H]1Br</smiles>

* : by asymmetric methods
Scheme 39 Comparison of substrate-controlled and asymmetric syntheses of (-)-isolaurallene (77)

When I gave lecture presentations, I would compare our substrate-controlled approach ${ }^{47}$ with the asymmetric strategy used by the Crimmins group, as illustrated in Scheme $39,{ }^{46 a, b}$ and would claim that our entirely substrate-based approach enabled us to achieve the more concise synthesis. Now, I spy Professor Scott Snyder of Columbia University over my shoulder as he describes syntheses of the (our!) target natural products laurefucin $(\mathbf{5 7})^{29}$ and pinnatifidenyne $(\mathbf{6 1})^{21}$ (see Schemes 23 and 25, respectively)<smiles>C=CCC(O[C@H](CC)C(=O)N(C)C)C(CC)OCC</smiles>

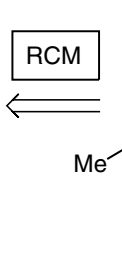

$\| \begin{gathered}\text { inter- } \\ \text { molecular } \\ \text { AEA }\end{gathered}$<smiles>C=CCC(OCC)C(CC)OCC(=O)N(C)C</smiles>
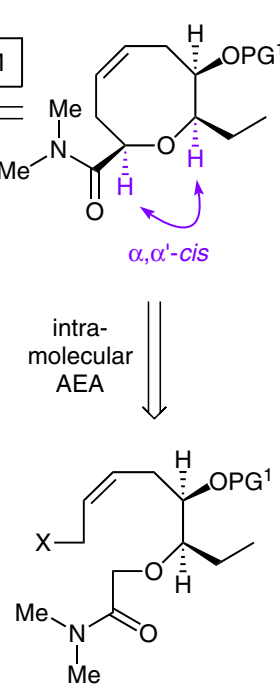

Me forbidden zone for RCM<smiles>CC(C)=CC[C@H]1O[C@@H]([C@H](C)O)[C@@H]2C(C)=CC[C@H](C(C)C)[C@@H]21</smiles>

cladiella-6,11-dien3-ol (65)

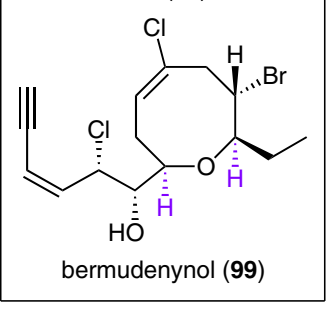

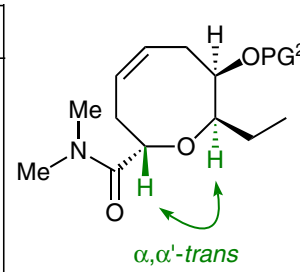

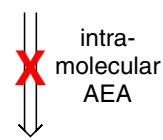<smiles>[X]C/C=C\C[C@H](O[R6])[C@@H](CC)OCC(=O)N(C)C</smiles>

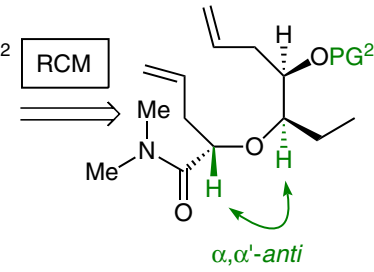

inter-
molecular
AEA<smiles>C=CC[C@H](O[Co])[C@@H](CC)OCC(=O)N(C)C</smiles>

Scheme 38 Ring-closing metathesis versus intramolecular amide enolate alkylation 
which employ a conceptually different strategy and occur in appreciably fewer steps. ${ }^{60}$ On the plus side, I take heart in this, in that it proves to me that the field of synthesis is alive and well. It will continue to be so, and some day someone will be gaining on him too!

When I started my independent research career 30 years ago, I encouraged my graduate students, mostly master's students, to go to the United States for a Ph.D. degree. The way I operated with these students was that I gave them a natural product synthesis project to work on to teach them experimental skills and give them a working knowledge of stereo-, regio-, and chemoselectivity. This prepared them so that they could pursue their doctoral degree in the USA with a competitive edge and minimal time needed for orientation. I took Ph.D. students only when they were not in a position to go abroad for some reason, and never had traditional postdoctorates. Shown in Figure 4 are some of the natural products we have synthesized in a substrate-controlled fashion under relatively spartan conditions with these talented students to whom I am very grateful.

Along with describing the adventures in synthesis that make up my career, I hope this account will make apparent the substantial influence my postdoctoral advisor Gilbert Stork has had on me. I have attempted to preach his chemistry, and moreover his approach to synthesis, as best as I can in Korea. Each time my efforts have been recognized in some small way, for instance by receiving the invitation to the 2008 Natural Products GRC and the 2011 Korean Academy of Science and Technology Award, I confess that the first thought to cross my mind is that if he was able to take crude starting material such as myself and convert me into a final product to be honored in this way, how great his tutelage and masterful mentoring must have been.

When I gave a lecture at the 2008 Natural Products GRC, one of the regular attendants asked me whether I had planned all of our syntheses. I told him, "Our syntheses are irrational but we have all the time in the world back in Korea. If we work hard, we can always come up with a solution. When we publish the whole story at the very end, people see me as smart and invite me to speak at conferences." It was a rather impromptu stupid answer, but in a sense it was an honor to get that kind of question. However, the thing is that when I came back to Seoul, I realized I did not have all the time in the world, with my mandatory retirement looming. By the time this account appears in print, the vivid autumn foliage depicted in Figure 4 will be outside my former office and everywhere else in Seoul. I will be retired and will surely miss the good times we had chasing down these substrate-controlled syntheses.

\section{References}

(1) Ahn, S. H.; Kim, D.; Chun, M. W.; Chung, W. Tetrahedron Lett. 1986, 27, 943.

(2) Kim, D.; Kim, H. S. J. Org. Chem. 1987, 52, 4633.

(3) Kim, D.; Ahn, S. K.; Bae, H.; Choi, W. J.; Kim, H. S. Tetrahedron Lett. 1997, 38, 4437.

(4) Kim, D.; Kim, I. H. Tetrahedron Lett. 1997, 38, 415.

(5) Stork, G.; Leonia, N. J. US Patent 2793233, 1957; Chem. Abstr. 1958, 52, 441.

(6) Tokoroyama, T.; Tsukamoto, M.; Iio, H. Tetrahedron Lett. 1984, 25, 5067.

(7) Tokoroyama, T.; Kusaka, H. Can. J. Chem. 1996, 74, 2487.

(8) Stork, G.; Cohen, J. F. J. Am. Chem. Soc. 1974, 96, 5270.

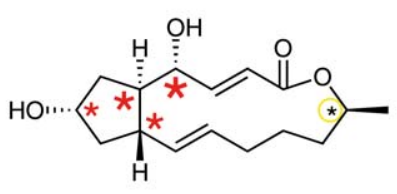

(+)-brefeldin A

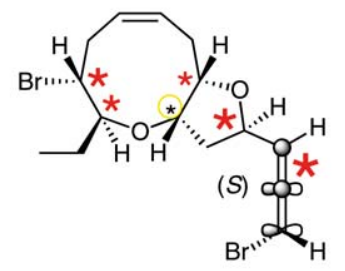

(+)-itomanallene $\mathrm{A}$

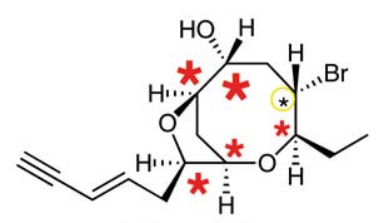

$(-)$-laurefucin

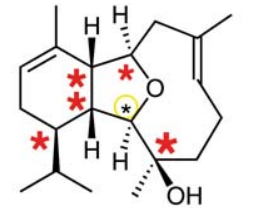

(-)-cladiella-6,11-dien-3-ol

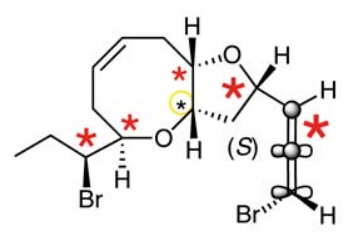

(+)-pannosallene

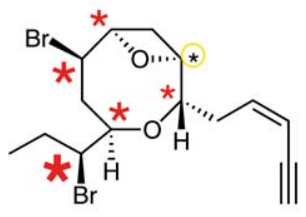

(+)-laureatin

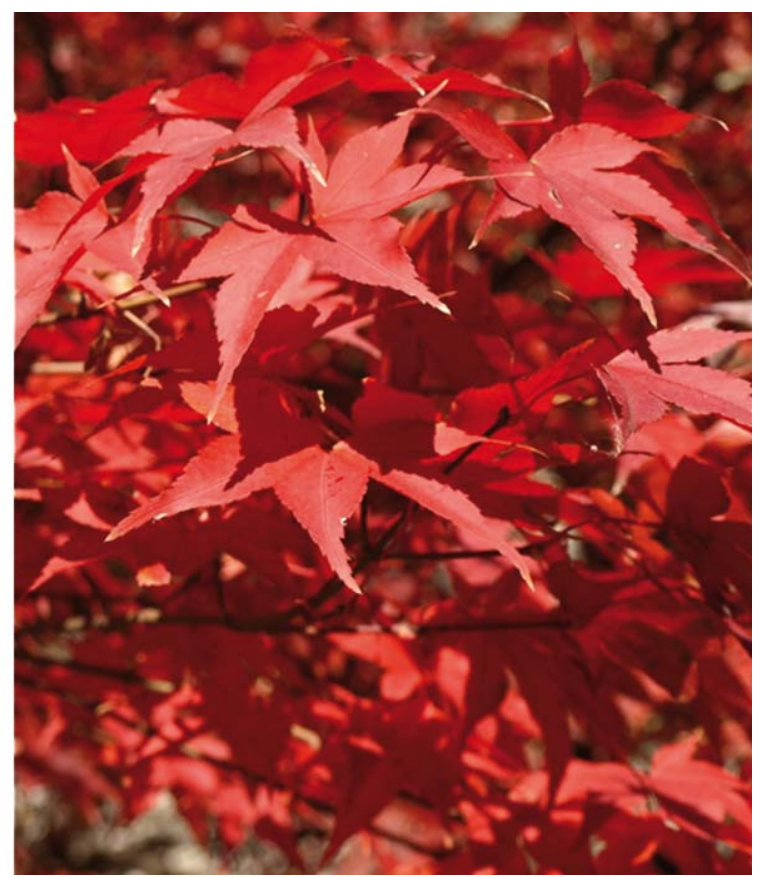

Figure 4 Some of the natural products synthesized in our laboratories using substrate-based control, where the red stars tell the story of the chiral centers present (left); a picture of beautiful autumn foliage with red star-shaped leaves, taken in 2008 outside my office where the strategies to create chiral centers were formulated (right) 
(9) (a) Kim, D.; Lee, Y. K.; Jang, Y. M.; Kim, I. O.; Park, S. W. J. Chem. Soc., Perkin Trans. 1 1990, 3221. (b) Kim, D.; Kwak, Y. S.; Shin, K. J. Tetrahedron Lett. 1994, 35, 9211.

(10) (a) Kim, D.; Lim, J. I. Tetrahedron Lett. 1995, 36, 5035. (b) Kim, D.; Lee, J.; Shim, P. J.; Lim, J. I.; Jo, H.; Kim, S. J. Org. Chem. 2002, 67, 764. (c) Kim, D.; Lee, J.; Shim, P. J.; Lim, J. I.; Doi, T.; Kim, S. J. Org. Chem. 2002, 67, 772.

(11) Kitahara, T.; Mori, K. Tetrahedron 1984, 40, 2935.

(12) Bartlett, P. A.; Green, F. R. III. J. Am. Chem. Soc. 1978, 100, 4858.

(13) Stork, G.; Raucher, S. J. Am. Chem. Soc. 1976, 98, 1583.

(14) Hoffmann, R. W.; Kemper, B. Tetrahedron Lett. 1982, 23, 845.

(15) For a recent review on intramolecular $\mathrm{S}_{\mathrm{N}} 2^{\prime}$ reactions, see: Devambatla, R. K. V.; Velagaleti, R.; Yarravarapu, N.; Fleming, F. F. Tetrahedron 2012, 68, 2925.

(16) Kim, D.; Lim, J. I.; Shin, K. J.; Kim, H. S. Tetrahedron Lett. 1993, 34, 6557.

(17) Jo, H.; Lee, J.; Kim, H.; Kim, S.; Kim, D. Tetrahedron Lett. 2003, 44, 7043.

(18) Kim, D.; Kim, H. S.; Yoo, J. Y. Tetrahedron Lett. 1991, 32, 1577.

(19) Kim, D.; Choi, W. J.; Hong, J. Y.; Park, I. Y.; Kim, Y. B. Tetrahedron Lett. 1996, 37, 1433.

(20) Choi, W. J.; Kim, D. unpublished results.

(21) Kim, H.; Choi, W. J.; Jung, J.; Kim, S.; Kim, D. J. Am. Chem. Soc. 2003, 125, 10238.

(22) Smith, A. B. III.; Empfield, J. R. Chem. Pharm. Bull. 1999, 47, 1671.

(23) Baek, S.; Jo, H.; Kim, H.; Kim, H.; Kim, S.; Kim, D. Org. Lett. 2005, 7, 75.

(24) (a) Evans, D. A.; Ennis, M. D.; Mathre, D. J. J. Am. Chem. Soc. 1982, 104, 1737. (b) Crimmins, M. T.; Emmitte, K. A.; Katz, J. D. Org. Lett. 2000, 2, 2165.

(25) Burke, S. D.; Deaton, D. N.; Olsen, R. J.; Armistead, D. M.; Blough, B. E. Tetrahedron Lett. 1987, 28, 3905.

(26) Nahm, S.; Weinreb, S. M. Tetrahedron Lett. 1981, 22, 3815.

(27) (a) Iida, H.; Yamazaki, N.; Kibayashi, C. J. Org. Chem. 1986, 51, 3769. (b) Clark, J. S.; Holmes, A. B. Tetrahedron Lett. 1988, 29, 4333.

(28) (a) Dyson, B. S.; Burton, J. W.; Sohn, T.; Kim, B.; Bae, H.; Kim, D. J. Am. Chem. Soc. 2012, 134, 11781; and references cited therein. (b) Hooz, J.; Gilani, S. S. H. Can. J. Chem. 1968, 46, 86.

(29) Kim, B.; Lee, M.; Kim, M. J.; Lee, H.; Kim, S.; Kim, D.; Koh, M.; Park, S. B.; Shin, K. J. J. Am. Chem. Soc. 2008, $130,16807$.

(30) Sohn, T.; Kim, M. J.; Kim, D. J. Am. Chem. Soc. 2010, 132, 12226 .

(31) For a review on the total synthesis of C-2-C-11 cyclized cembranoids, see: Ellis, J. M.; Crimmins, M. T. Chem. Rev. 2008, 108, 5278 .

(32) (a) Crimmins, M. T.; Brown, B. H. J. Am. Chem. Soc. 2004, 126, 10264. (b) Crimmins, M. T.; Brown, B. H.; Plake, H. R. J. Am. Chem. Soc. 2006, 128, 1371.

(33) Kim, H.; Lee, H.; Kim, J.; Kim, S.; Kim, D. J. Am. Chem. Soc. 2006, 128, 15851

(34) Bernardelli, P.; Moradei, O. M.; Friedrich, D.; Yang, J.; Gallou, F.; Dyck, B. P.; Doskotch, R. W.; Lange, T.; Paquette, L. A. J. Am. Chem. Soc. 2001, 123, 9021.

(35) Barrett, A. G. M.; Godfrey, C. R. A.; Hollinshead, D. M.; Prokopiou, P. A.; Barton, D. H. R.; Boar, R. B.; Joukhadar, L.; McGhie, J. F.; Misra, S. C. J. Chem. Soc., Perkin Trans. 1 1981, 1501 .
(36) (a) Clark, J. S.; Berger, R.; Hayes, S. T.; Thomas, L. H.; Morrison, A. J.; Gobbi, L. Angew. Chem. Int. Ed. 2010, 49, 9867. (b) Clark, J. S.; Berger, R.; Hayes, S. T.; Senn, H. M.; Farrugia, L. J.; Thomas, L. H.; Morrison, A. J.; Gobbi, L. J. Org. Chem. 2013, 78, 673.

(37) For a successful application that is a notable exception, see: Kim, H.; Lee, H.; Lee, D.; Kim, S.; Kim, D. J. Am. Chem. Soc. 2007, 129, 2269.

(38) Suzuki, M.; Takahashi, Y.; Mitome, Y.; Itoh, T.; Abe, T.; Masuda, M. Phytochemistry 2002, 60, 861.

(39) Kurata, K.; Furusaki, A.; Suehiro, K.; Katayama, C.; Suzuki, T. Chem. Lett. 1982, 1031.

(40) Suzuki, M.; Kurosawa, E.; Furusaki, A.; Katsuragi, S.; Matsumoto, T. Chem. Lett. 1984, 1033.

(41) Lowe, G. Chem. Commun. 1965, 411.

(42) Jeong, W.; Kim, M. J.; Kim, H.; Kim, S.; Kim, D.; Shin, K. J. Angew. Chem. Int. Ed. 2010, 49, 752.

(43) (a) Gaunt, M. J.; Hook, D. F.; Tanner, H. R.; Ley, S. V. Org. Lett. 2003, 5, 4815. (b) Lainé, D.; Fujita, M.; Ley, S. V. J. Chem. Soc., Perkin Trans. 1 1999, 1639.

(44) Keck, G. E.; Boden, E. P. Tetrahedron Lett. 1984, 25, 265.

(45) Kim, B.; Cheon, G.; Park, J.; Lee, H.; Kim, H.; Kim, S.; Kim, D. Heterocycles 2007, 74, 171

(46) (a) Crimmins, M. T.; Emmitte, K. A. J. Am. Chem. Soc. 2001, 123, 1533. (b) Crimmins, M. T.; Emmitte, K. A.; Choy, A. L. Tetrahedron 2002, 58, 1817. (c) Crimmins, M. T.; Powell, M. T. J. Am. Chem. Soc. 2003, 125, 7592.

(47) Kim, M. J.; Sohn, T.; Kim, D.; Paton, R. S. J. Am. Chem. Soc. 2012, 134, 20178.

(48) Kim, S.; Ahn, K. H. J. Org. Chem. 1984, 49, 1717.

(49) Grese, T. A.; Hutchinson, K. D.; Overman, L. E. J. Org. Chem. 1993, 58, 2468.

(50) (a) Stork, G.; Gardner, J. O.; Boeckman, R. K. Jr.; Parker, K. A. J. Am. Chem. Soc. 1973, 95, 2014. (b) Stork, G.; Boeckman, R. K. Jr. J. Am. Chem. Soc. 1973, 95, 2016.

(51) For recent reviews on nitrile anion cyclizations, see: (a) Fleming, F. F.; Wei, Y.; Liu, W.; Zhang, Z. Tetrahedron 2008, 64, 7477. (b) Fleming, F. F.; Gudipati, S. Eur. J. Org. Chem. 2008, 5365.

(52) For details of this intramolecular nitrile anion alkylation $\left(\mathbf{9 0}^{\prime}\right.$ $\rightarrow$ 91'), see: (a) Jeong, W. Ph.D. Thesis; Seoul National University: Republic of Korea, 2010, the results are comparable to those reported in connection with our isolaurallene synthesis: (b) See also ref. 47.

(53) Park, J.; Kim, B.; Kim, H.; Kim, S.; Kim, D. Angew. Chem. Int. Ed. 2007, 46, 4726.

(54) Fukuzawa, A.; Kurosawa, E. Tetrahedron Lett. 1979, 20, 2797.

(55) Lyakhova, E. G.; Kalinovsky, A. I.; Dmitrenok, A. S.; Kolesnikova, S. A.; Fedorov, S. N.; Vaskovsky, V. E.; Stonik, V. A. Tetrahedron Lett. 2006, 47, 6549.

(56) Suzuki, M.; Takahashi, Y.; Matsuo, Y.; Masuda, M. Phytochemistry 1996, 41, 1101.

(57) The two ratios in parentheses for the Overman protocol in Schemes 35-37 represent the results from the titanium (trimethylsilyl)acetylide addition and copper-catalyzed anti$\mathrm{S}_{\mathrm{N}} 2^{\prime}$ reaction, respectively.

(58) Kim, G.; Sohn, T.; Kim, D.; Paton, R. S. Angew. Chem. Int. Ed., doi: 10.1002/anie.201308077.

(59) Chao, W.; Weinreb, S. M. Org. Lett. 2003, 5, 2505.

(60) Snyder, S. A.; Brucks, A. P.; Treitler, D. S.; Moga, I. J. Am. Chem. Soc. 2012, 134, 17714. 